Chapman University

Chapman University Digital Commons

Mathematics, Physics, and Computer Science

Faculty Articles and Research

Science and Technology Faculty Articles and

Research

$9-27-2021$

\title{
Atomistic Simulations and In Silico Mutational Profiling of Protein Stability and Binding in the SARS-CoV-2 Spike Protein Complexes with Nanobodies: Molecular Determinants of Mutational Escape Mechanisms
}

Gennady M. Verkhivker

Steve Agajanian

Deniz Yasar Oztas

Grace Gupta

Follow this and additional works at: https://digitalcommons.chapman.edu/scs_articles

Part of the Amino Acids, Peptides, and Proteins Commons, Epidemiology Commons, Medical Biochemistry Commons, Medicinal-Pharmaceutical Chemistry Commons, Other Chemicals and Drugs Commons, Other Chemistry Commons, and the Virus Diseases Commons 
Atomistic Simulations and In Silico Mutational Profiling of Protein Stability and Binding in the SARS-CoV-2 Spike Protein Complexes with Nanobodies: Molecular Determinants of Mutational Escape Mechanisms

\section{Comments}

This article was originally published in ACS Omega in 2021. https://doi.org/10.1021/acsomega.1c03558

This scholarship is part of the Chapman University COVID-19 Archives.

Creative Commons License

(c) $(1) \Theta$

This work is licensed under a Creative Commons Attribution-Noncommercial-No Derivative Works 4.0 License.

Copyright

The authors 


\title{
Atomistic Simulations and In Silico Mutational Profiling of Protein Stability and Binding in the SARS-CoV-2 Spike Protein Complexes with Nanobodies: Molecular Determinants of Mutational Escape Mechanisms
}

\author{
Gennady M. Verkhivker,* Steve Agajanian, Deniz Yasar Oztas, and Grace Gupta \\ Cite This: https://doi.org/10.1021/acsomega.1c03558 \\ Read Online
}

ACCESS

Wlll Metrics \& More

回 Article Recommendations

Supporting Information

ABSTRACT: Structure-functional studies have recently revealed a spectrum of diverse high-affinity nanobodies with efficient neutralizing capacity against SARS-CoV-2 virus and resilience against mutational escape. In this study, we combine atomistic simulations with the ensemble-based mutational profiling of binding for the SARS-CoV-2 S-RBD complexes with a wide range of nanobodies to identify dynamic and binding affinity fingerprints and characterize the energetic determinants of nanobody-escaping mutations. Using an in silico mutational profiling approach for probing the protein stability and binding, we examine dynamics and energetics of the SARS-CoV-2 complexes with single nanobodies $\mathrm{Nb6}$ and $\mathrm{Nb20}, \mathrm{VHH} \mathrm{E}$, a pair combination $\mathrm{VHH} \mathrm{E}+\mathrm{U}$, a biparatopic nanobody VHH VE, and a combination of the CC12.3
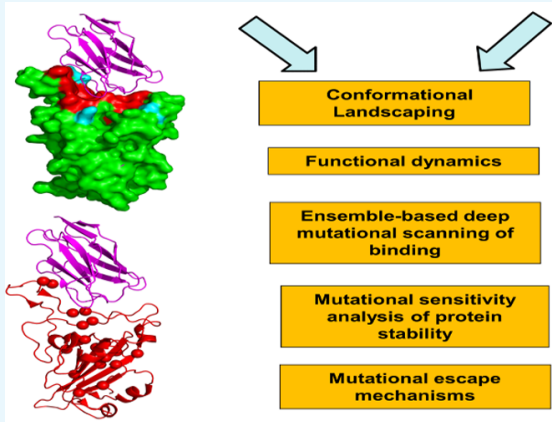
analysis of protein stability
stats

Mutational escape utational escap
mechanisms

In Silico Mutational Scanning of Binding and Stabilit in the SARS-CoV-2 Spike Complexes with Nanobodies antibody and $\mathrm{VHH} \mathrm{V} / \mathrm{W}$ nanobodies. This study characterizes the binding energy hotspots in the SARS-CoV-2 protein and complexes with nanobodies providing a quantitative analysis of the effects of circulating variants and escaping mutations on binding that is consistent with a broad range of biochemical experiments. The results suggest that mutational escape may be controlled through structurally adaptable binding hotspots in the receptor-accessible binding epitope that are dynamically coupled to the stability centers in the distant binding epitope targeted by $\mathrm{VHH} \mathrm{U} / \mathrm{V} / \mathrm{W}$ nanobodies. This study offers a plausible mechanism in which through cooperative dynamic changes, nanobody combinations and biparatopic nanobodies can elicit the increased binding affinity response and yield resilience to common escape mutants.

\section{INTRODUCTION}

Structural and functional studies of the SARS-CoV-2 infection mechanisms have been largely focused on the viral spike (S) glycoprotein that interacts with the host cell receptor ACE2 and produces a cascade of conformational changes that lead to membrane fusion. ${ }^{1-3}$ The recent growth of cryo-EM structures for the SARS-CoV-2 S proteins showed a remarkable diversity of conformational changes of the $S$ protein trimers in which $S 1$ and S2 subunits can act synchronously to orchestrate a delicate balance of stability and dynamics that governs functional rearrangements of the prefusion and postfusion forms. ${ }^{4,5}$ The conformational changes of the $S$ protein trimers associated with binding to ACE2 are executed through spontaneous thermal movements of the $\mathrm{N}$-terminal domain and the receptor-binding domain ( $\mathrm{RBD}$ ) which fluctuates between the RBD-down (closed) and the open RBD-up form. ${ }^{6-19}$ Functional and conformational plasticity of SARS-CoV-2 S proteins and their ability to produce specific and precise protein responses to binding have been revealed in the rapidly expanding number of cryo-EM and $\mathrm{X}$-ray spike complexes with a wide range of neutralizing antibodies. ${ }^{20-28}$ These studies have classified SARS-CoV-2 antibodies into several major categories that prominently feature monoclonal antibodies that target the receptor binding motif (RBM) region of the RBD epitope. $^{28-31}$ The recent structure-functional studies of the SARS-CoV-2 $S$ protein binding revealed the binding epitopes and clarified basic recognition mechanisms for a number of highly potent antibodies targeting the RBM region, which include REGN10933, B38, CB6, CC12.3, and BD-236 antibodies. $^{32-38}$ These pioneering studies also demonstrated that antibody cocktails targeting different binding epitopes could produce strong synergistic effects, leading to the stronger neutralization profile and the improved resilience against

Received: July 6, 2021

Accepted: September 10, 2021 
antibody-induced mutations. Biochemical studies using deep mutagenesis experiments have enabled comprehensive functional mappings of mutational responses and characterized patterns of mutational escape to many antibodies. ${ }^{39-42}$ These illuminating experiments revealed that escape-resistant antibody cocktails can impose conformation-specific "breaks" on the plasticity of the $S$ protein and significantly restrict virus "creativity" in finding the sites of immune escape that would not deleteriously affect the protein stability and ACE2 binding. ${ }^{39-42}$ A comprehensive review of the SARS-CoV-2 S mutational variants and mechanisms of immune escape dissected the evolutionary and structural origins underlying the impacts of mutations on antigenicity. ${ }^{43}$ This study suggested several distinct mechanisms by which mutations can alter the antigenic responses and elicit antibody evasion, which included mutation-induced alteration of the binding epitopes for antibodies, the increasing host receptor binding avidity, changes in the glycosylation environment, and also allosterically regulated structural and dynamic changes in the spike protein. ${ }^{43}$ The global circulating mutants of the SARSCoV-2 S protein discovered through epidemiological surveillance are often prominently featured in a wide range of antibody-escaping mutational patterns. ${ }^{44-54}$ Many of these circulating mutations have also been identified in deep mutagenesis mapping experiments as escaping variants to a wide range of highly potent class I and class II antibodies, suggesting that virus can often evolve to evade antibody binding by targeting flexible RBD regions sensitive to binding. ${ }^{39-42}$

Nanobodies have shown an increasing promise in combating escaping mutations. ${ }^{55-57}$ An ultrapotent nanobody $\mathrm{Nb6}$ neutralizes SARS-CoV-2 by stabilizing the closed form of the $S$ protein and inhibiting binding with the ACE2 receptor. ${ }^{55}$ Another group of high-affinity nanobodies including Nb20 and $\mathrm{Nb} 21$ can neutralize SARS-CoV-2 by targeting a dominant epitope that overlaps with the ACE2 binding site. ${ }^{56}$ A number of highly potent neutralizing nanobodies that resist circulating variants of SARS-CoV-2 by targeting existing and novel epitopes were recently unveiled. ${ }^{57}$ The cryo-EM structures for different classes of nanobodies reported in this study suggested the mechanisms of high affinity and neutralizing activity that are based on the synergistic exploitation of distinct epitopes using binding avidity effects. ${ }^{57}$ Another diverse group of high-affinity nanobodies with an excellent neutralizing activity against SARS-CoV-2 $\mathrm{S}$ protein that can effectively combat resistance to common escape mutants featured complementary binding to the nonoverlapping epitopes in the RBD core and a more flexible RBM region. ${ }^{58}$ These studies suggested that multimeric nanobody combinations can significantly improve binding affinity via positive cooperativity between binding sites that can drastically limit virus "options" to find alternative mutational sites and allow for the reduction of susceptibility to escape mutations. ${ }^{58}$

Protein engineering studies discovered several unique $\mathrm{VH}$ binders that recognize two separate epitopes within the ACE2 binding interface with nanomolar affinity, leading to multivalent and biparatopic $\mathrm{VH}$ constructs with the markedly increased affinity and neutralization potency to the SARS-CoV2 virus as compared to the standalone $\mathrm{VH}$ domain. ${ }^{59}$ Biochemical saturation mutagenesis and fluorescence-activated cell sorting for mutant screening mapped patterns of escape mutants for some of these nanobodies, indicating that vulnerable sites were localized on the periphery of the
ACE2-binding site. ${ }^{60}$ A number of recently discovered nanobodies with efficient neutralizing capacity and resilience against mutational escape included $\mathrm{VHH}$ E nanobody targeting the RBM-binding epitope and VHHs $\mathrm{U}, \mathrm{V}$, and $\mathrm{W}$ nanobodies that bind to a distant cryptic epitope in the RBD. ${ }^{61-63}$ This structure-functional "tour de force" investigation provided a compelling evidence that synergistic cocktails of nanobodies interacting with distinct RBD epitopes can efficiently suppress the emergence of escape mutants that were resistant to individual nanobodies. ${ }^{61}$ Of particular interest were biparatopic VHH EV and VE nanobodies that were superior in preventing mutual escape as compared to both individual nanobodies and pair combinations $\mathrm{VHH} \mathrm{E}+\mathrm{U}, \mathrm{VHH} \mathrm{E}+\mathrm{V}$, and $\mathrm{VHH} \mathrm{E}+$ W. ${ }^{61}$ The two highly potent nanobodies H11-D4 and H11$\mathrm{H} 4$ recognize the same epitope that is immediately adjacent to and partly overlapping with the ACE2-binding region. ${ }^{64}$ The high-affinity nanobodies and nanobody cocktails consisting of two noncompeting nanobodies can potently neutralize both wild-type SARS-CoV-2 and the N501/D614G variants. ${ }^{65}$ Neutralization of SARS-CoV-2 by low-picomolar, hyperthermostable, and mutation-tolerant $\mathrm{VHH}$ nanobodies was recently reported, unveiling the cryo-EM structures of a ternary complex of the RBD with the fold-promoting nanobody Re9F06 and the potently neutralizing Re5D06 nanobody that bind synergistically to the opposite sides of the RBD and occlude ACE binding. ${ }^{66}$ The tandem fusion of the Re5D06 nanobody binding to the RBM region and the hyperthermostable Re9F06 nanobody that binds to the RBD core epitope produced a binding avidity effect unaffected by immune-escape mutants $\mathrm{K} 417 \mathrm{~N} / \mathrm{T}, \mathrm{E} 484 \mathrm{~K}, \mathrm{~N} 501 \mathrm{Y}$, and L452R. ${ }^{66}$ The recent fascinating discovery of nanobodies and nanobody cocktails from camelid mice and llamas that neutralize SARS-CoV-2 variants showed a remarkable ability of multivalent nanobodies to combat escaping mutations through synchronized avidity between binding epitopes. ${ }^{67}$ While a highly potent group of llama nanobodies ( $\mathrm{Nb} 17$, $\mathrm{Nb} 19$, and $\mathrm{Nb56}$ ) directly target the ACE2-binding interface, picomolar nanobodies from nanomouse ( $\mathrm{Nb} 12$ and $\mathrm{Nb30}$ ) bind to a previously unappreciated conserved RBD epitope outside of the ACE2-binding motif which is not accessible to human antibodies, allowing for the mitigation of adverse effects caused by escape mutations at E484 and N501 positions. ${ }^{67}$ These studies suggested that the biparatopic nanobodies can be superior to monoclonal antibodies, single nanobodies, and even conventional nanobody cocktails in combating escape mutations by effectively leveraging binding avidity and allosteric cooperativity mechanisms coupled with the reduced entropic cost of binding to distinct epitopes.

Computer simulations have made important contributions to our understanding of the dynamics and the function of SARS-CoV-2 glycoproteins. ${ }^{68-76}$ The atomistic models of a full-length SARS-CoV-2 $S$ protein in a viral membrane allowed for the simulation studies of the SARS-CoV-2 $S$ trimer structures in a glycosylated environment. ${ }^{69}$ All-atom molecular dynamics (MD) simulations of the full-length SARS-CoV-2 S glycoprotein embedded in the viral membrane, with a complete glycosylation profile, provided significant structural insights into functional spike conformations and mechanisms of transitions ${ }^{70-73}$ Multimicrosecond MD simulations with four full-length fully glycosylated $S$ proteins characterized structural and dynamic signatures of antibodies and vaccine epitopes. $^{74} \mathrm{We}$ integrated coarse-grained and atomistic MD simulations with network modeling to reveal that the SARS- 
CoV-2 S proteins can function as allosterically regulated dynamic machines that exploit the plasticity of functional regions exposed to binding with ACE2 and interacting proteins to fine-tune differential responses to antibody binding. ${ }^{77-82}$ These studies showed that examining the allosteric behavior of the SARS-CoV-2 S proteins may be useful to rationalize the growing body of diverse experimental data. A critical review of computational studies of the SARS-CoV-2 S proteins highlighted the synergies between experiments and simulations, outlining directions for computational biology research in understanding the mechanisms of COVID-19 protein targets. ${ }^{83}$

In this study, we combine atomistic simulations with the ensemble-based mutational profiling of binding of the SARSCoV-2 S-RBD complexes with a wide range of nanobodies to identify dynamic and binding affinity fingerprints and characterize the energetic determinants of nanobody-escaping mutations. Using an in silico mutational profiling approach for probing the protein stability and binding, we examine dynamics and energetics of the SARS-CoV- 2 complexes with single nanobodies $\mathrm{Nb} 6$ and $\mathrm{Nb} 20, \mathrm{VHH}$, a pair combination $\mathrm{VHH} \mathrm{E}+\mathrm{U}$, a biparatopic nanobody $\mathrm{VHH} \mathrm{VE}$, and a combination of the CC12.3 antibody and $\mathrm{VHH} \mathrm{V/W}$ nanobodies. This study characterizes the binding energy hotspots in the SARS-CoV-2 protein and complexes with nanobodies providing a quantitative analysis of the effects of circulating variants and escaping mutations on binding that is consistent with a broad range of biochemical experiments. Our results suggest a plausible mechanism in which nanobody combinations and biparatopic nanobodies can elicit an increased binding affinity response and yield resilience to common escape mutants.

\section{RESULTS AND DISCUSSION}

Conformational Dynamics and Distance Fluctuation Stability Analysis of the SARS-CoV-2 S RBD Complexes Reveal Nanobody-Induced Modulation of Spike Plasticity. MD simulations of the SARS-CoV-2 S-RBD protein complexes with a panel of nanobodies (Figure 1) examined how nanobody binding targeting distinct binding epitopes can change the dynamics of the RBD regions. A comparative analysis of the conformational flexibility profiles revealed nanobody-induced stabilization of the interacting regions, especially in the complex with the biparatopic nanobody $\mathrm{VHH}$ VE (Figure 2A). The RBD core regions including the $\alpha$-helical segments of the RBD (residues 349-353, 405-410, and 416423 ) and central $\beta$ strands (residues 354-363, 389-405, and 423-436) showed only very small thermal fluctuations (Figure $2 A$, Supporting Information, Figure S1). Interestingly, the antiparallel $\beta$-sheets $\beta 5$ and $\beta 6$ (residues $451-454$ and $491-$ 495) also showed an increased stabilization. These structural elements of the RBD are involved in linking the central core with the more flexible RBM region and could serve as important anchors of dynamic communication between structurally stable and dynamic RBD region fluctuations (Supporting Information, Figure S1). The stabilization of these regions in the SARS-CoV-2 S complexes is particularly important due to structural proximity to the sites of common mutational escape L452, F486, and F490 (Supporting Information, Figures S1 and S2). Conformational dynamic profiles reaffirmed the stability of the $\alpha$-helical segments in the RBD that are located near the cryptic binding epitope (residues 369-384) targeted by $\mathrm{VHH} \mathrm{U} / \mathrm{V} / \mathrm{W}$ nanobodies (Supporting Information, Figures S2 and S3). These residues
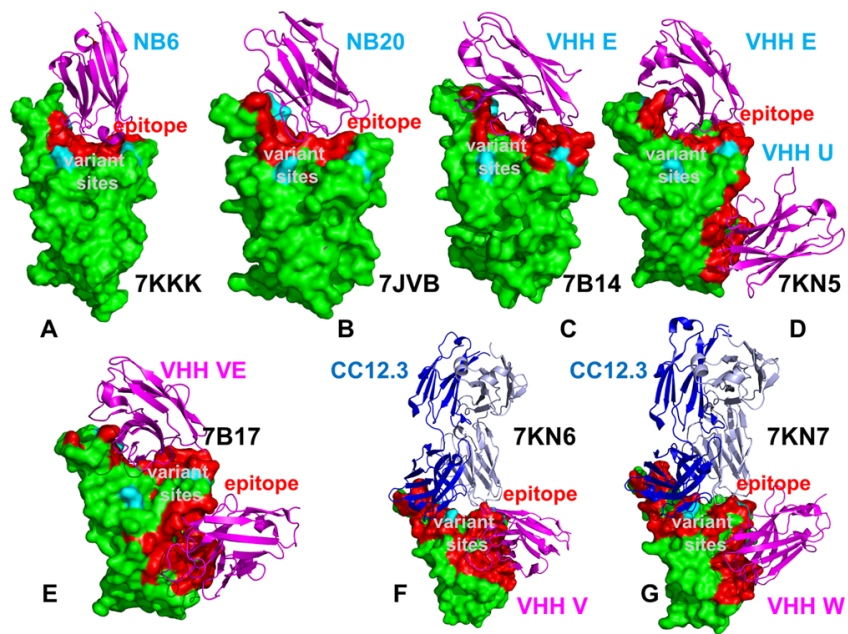

Figure 1. Crystal structures of the SARS-CoV-2 $\mathrm{S}$ trimer structures used in this study included complexes with $\mathrm{Nb6}$ (A), $\mathrm{Nb} 20$ (B), VHH $\mathrm{E}$ (C), a nanobody combination pair $\mathrm{VHH} \mathrm{E} / \mathrm{VHH} \mathrm{U}$ (D), biparatopic nanobody VHH VE (E), CC12.3/VHH V pair (F), and a CC12.3/VHH W pair (G). The S-RBD are in green surfaces. The binding epitope residues are in the red surface. The bound nanobodies are in magenta ribbons and annotated. The heavy chain of CC12.3 is in blue color and light chain in cyan color. The sites K417, L452, E484, and N501 subjected to circulating mutational variants are shown in the cyan surface and annotated.

provide a stable anchoring platform for nanobodies binding to the cryptic epitope, allowing for the modulation of RBD flexibility and optimization of binding interactions with the more dynamic RBD-binding epitope (Figure 2). The S-RBD region also contains eight conserved cysteine residues, six of which form three disulfide linkages (C336-C361, C379C432, and C391-C525), which provide support to the $\beta$-sheet structure in the S-RBD core (Supporting Information, Figures S2 and S3). Conformational dynamics of the biparatopic nanobody complex VHH VE showed a significant stabilization of the $S-R B D$ regions and displayed the markedly reduced fluctuations in the flexible RBM regions that become more rigid and contribute to the binding interactions (Figure 2A). The important functional sites E484, F486, and N501 belong to flexible RBM regions but through binding contacts with nanobodies become more stable in the complexes (Supporting Information, Figures $\mathrm{S} 2$ and $2 \mathrm{C}-\mathrm{E}$ ). Interestingly, these positions are commonly shared sites of nanobody-escaping mutations and only the biparatopic nanobody showed resilience to mutational escape in these positions. At the same time, an appreciable level of flexibility was seen for the $S$ $\mathrm{RBD}$ complexes with $\mathrm{Nb} 20$ (Figure 2B) and $\mathrm{VHH}$ E (Figure $2 \mathrm{C}$ ) in regions that are distant from the RBM-binding epitope (residues 359-372 and 380-390).

Another RBD region that remained flexible in the SARSCoV-2 complexes with the nanobodies is the tip of the RBM loop (residues 473-483) (Figure 2). While the functional sites E484, F486, and N501 belong to the intrinsically mobile RBM regions, these residues are involved in binding contacts with nanobodies $\mathrm{Nb} 20$ and $\mathrm{VHH}$ E, leading to their stabilization in the complexes (Figure 2, Supporting Information, Figure S2). Of some importance is a differential stabilization of the N501 residue that retained some degrees of mobility in the S-RBD complexes with $\mathrm{Nb} 20$ (Figure 2C) and $\mathrm{VHH}$ E (Figure 2D) but exhibited an increased stabilization in the complex with the 


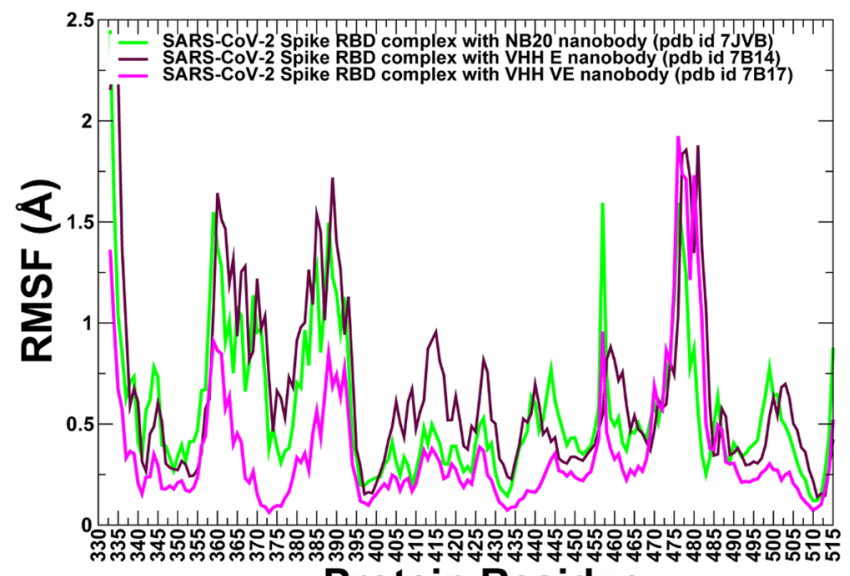

A

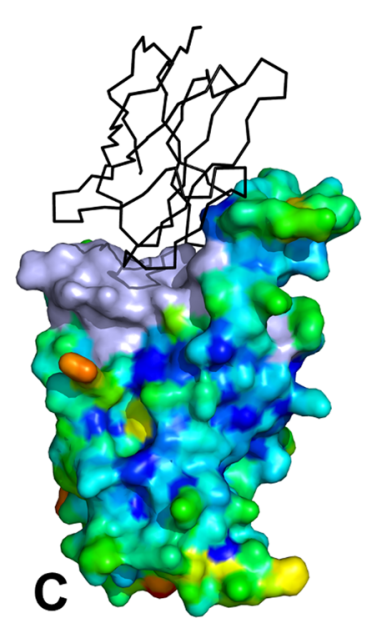

Protein Residue

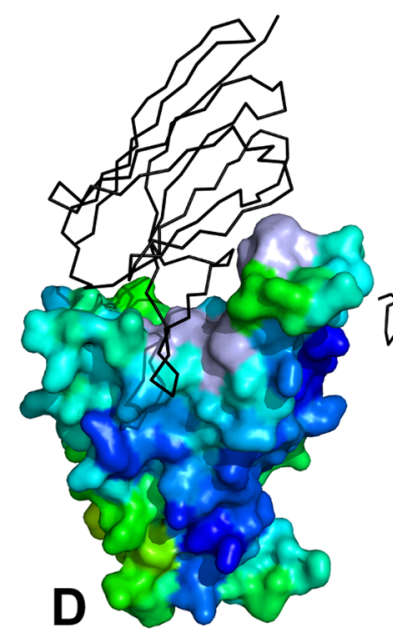

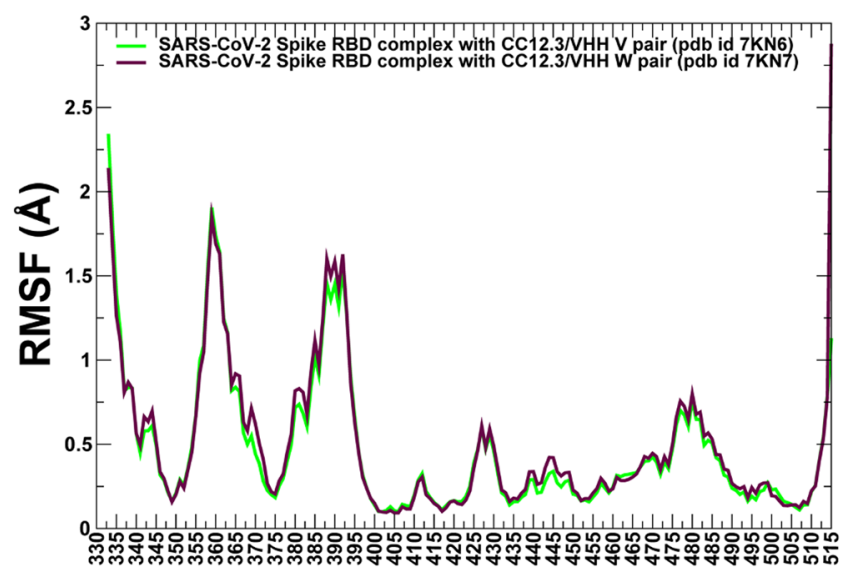

Protein Residue
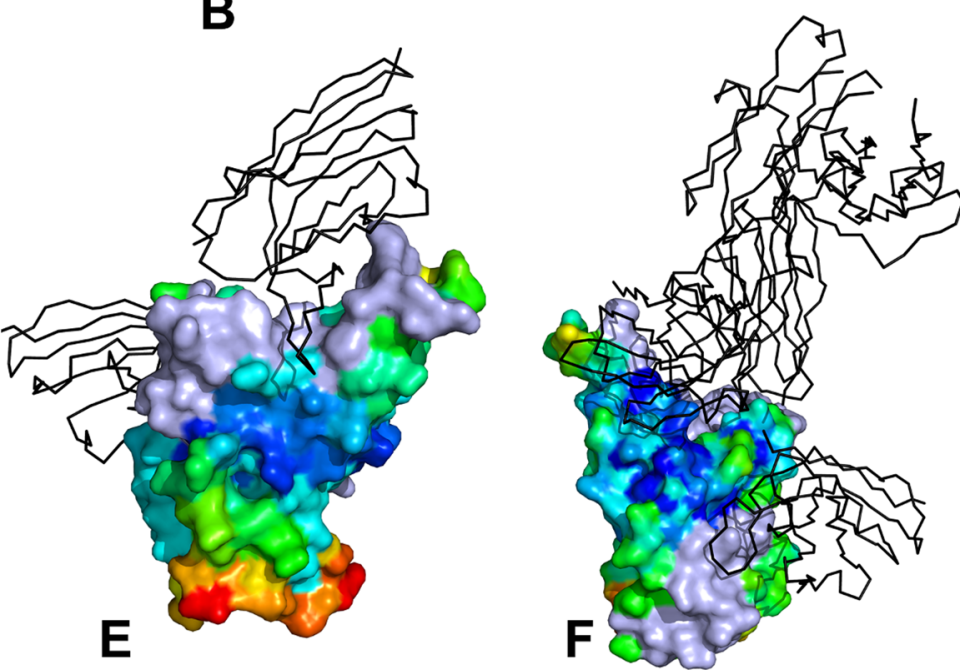

Figure 2. Conformational dynamics of the SARS-CoV-2 S-RBD. (A) Root-mean-square fluctuations (RMSFs) for MD trajectories of the SARSCoV-2 S-RBD complex with Nb20 (in green), VHH E (in maroon), and VHH VE (in magenta lines). (B) RMSF profile for the MD trajectories of $\mathrm{S}$-RBD binding with the CC12.3/VHH V pair (in green) and CC12.3/VHH W (in maroon). Conformational mobility maps for S-RBD complexes with $\mathrm{Nb} 20$ (C), VHH E (D), VHH VE (E), and CC12.3/VHH V (F). The mobility level is in a color-coded scale from rigid in blue to flexible in red. The nanobodies are shown in black-colored cartoons.

biparatopic nanobody VHH VE (Figure 2E). Structural maps of the conformational mobility profile also highlighted a generally observed reduction of mobility for regions proximal to the binding epitopes (Figure 2E, Supporting Information, Figure S2). Conformational dynamic profiles highlighted some interesting changes in protein mobility near the cryptic epitope targeted by $\mathrm{VHH} \mathrm{U} / \mathrm{V} / \mathrm{W}$ nanobodies (Figure $2 \mathrm{C}-\mathrm{F}$ ). We observed that the stability of this region increased in the $S$ RBD complex with a single VHH E nanobody that binds to a flexible RBM epitope. Hence, VHH E binding may allosterically modulate stability in the distant epitope (Figure 2C). This effect may facilitate couplings between distant epitopes and promote binding avidity in the SARS-CoV-2 complexes with $\mathrm{VHH} \mathrm{E} / \mathrm{VHH} \mathrm{U}$ combination and the VHH VE biparatopic nanobody.

The most common way for viruses to escape nanobody blockage is by mutating residues in the easily accessible regions where viruses can tolerate mutations while escaping immune challenge. Conformational dynamic analysis suggested that structural stabilization of the S-RBD regions induced by the VHH VE biparatopic nanobody can effectively suppress the mutational escape by reducing flexibility in the two remote epitopes, which may reduce the virus potential for adaptability in the functionally relevant for binding regions. A different pattern of dynamic changes was seen in the S-RBD complexes with $\mathrm{CC} 12.3 / \mathrm{VHH} \mathrm{V}$ and $\mathrm{CC} 12.3 / \mathrm{VHH} \mathrm{W}$ pairs (Figure $2 \mathrm{~B}$ ). In some contrast, the entire RBD interface showed a considerable stabilization owing to a dense network of specific interactions formed by the CC12.3 antibody. On the other hand, a very similar dynamic profile is seen in a second binding epitope targeted by $\mathrm{VHH} \mathrm{V}$ and $\mathrm{VHH}$ W nanobodies (Figure 2B). Of particular importance is the stability of binding interactions formed by the loop in the distal end of RBD (residues 473-488). The antibody-induced stability of the distal loop is specific for CC12.3 binding as opposed to $\mathrm{VHH}$ E- or $\mathrm{Nb20}$-binding interactions. Structural maps depicted the greater stabilization of the RBM region in the complex with the $\mathrm{CC} 12.3 / \mathrm{VHH} \mathrm{V}$ pair, as compared to other complexes, indicating stronger modulation of stability by the antibody binding (Figure 2E,F).

The correlation matrix of the S-RBD complex with VHH E showed that the S-RBD binding interface residues can be anticorrelated with the fluctuations of $\mathrm{VHH} \mathrm{E}$ residues (Supporting Information, Figure S4). In the complex with the VHH VE biparatopic nanobody, the S-RBD dynamic couplings are generally stronger and positively correlated with 


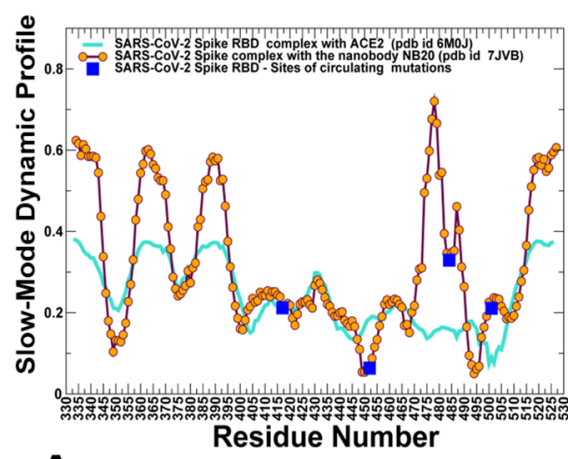

A
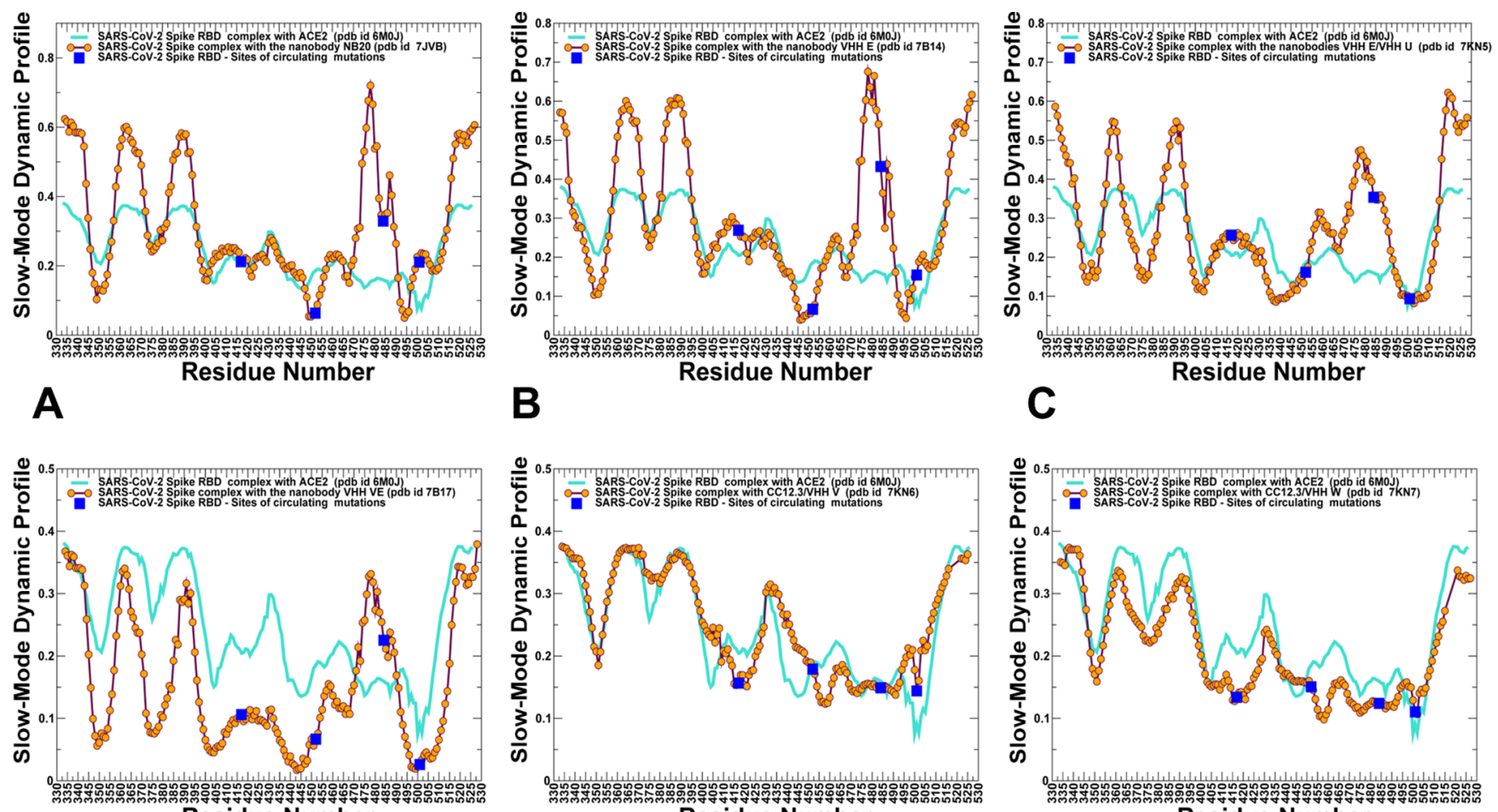

B

C
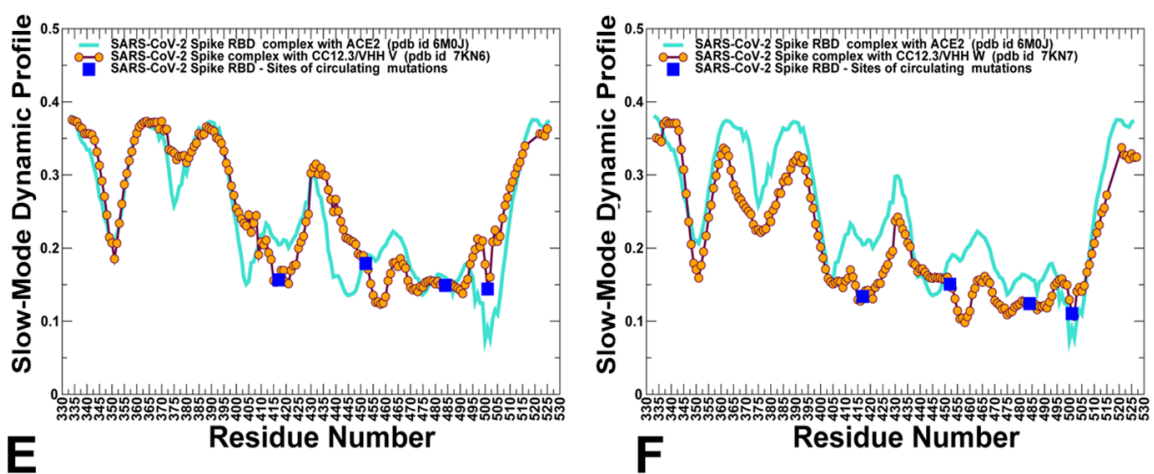

Figure 3. Collective dynamic profiles of the SARS-CoV-2 S complexes for Nb20 (A), VHH E (B), VHH E/VHH U (C), VHH VE (D), CC12.3/ VHH V (E), and CC12.3/VHH W (F). The profiles represent the mean-square displacements in functional motions averaged over the three lowest frequency modes. The slow-mode profiles are shown in maroon-colored lines with individual data points highlighted in filled orange circles. The slow-mode profile for the S-RBD complex with ACE2 is shown for comparison in turquoise color lines.

fluctuations of the VHH V at the cryptic site while mostly anticorrelated with VHH E (Supporting Information, Figure S4). The movements of VHH E and VHH V are dynamically strongly coupled and anticorrelated. The positive correlations between fluctuations of the S-RBD and VHH V residues could be seen for the complex with the CC12.3/VHH V complex, but weaker couplings were observed between S-RBD and CC12.3 (Supporting Information, Figure S4). The presence of strong positive correlations between S-RBD and $\mathrm{VHH} \mathrm{V}$ residues reflected a considerable stabilization of the binding interface at the cryptic epitope. Based on these observations, it is tempting to speculate that this epitope can act as a structural anchor and global hinge of functional movement in the complexes with the biparatopic nanobody VHH VE. The results suggest that the structural stability of the S-RBD induced by VHH VE may be linked to experimentally observed avidity effects when multiple interactions in different epitopes can synergize and enhance the strength of protein-protein interactions in the multivalent complex, thus providing the thermodynamic advantage for stronger binding and neutralization capacity. ${ }^{84-86}$

Modulation of Functional Dynamics by Nanobody Binding: Mutational Escape Sites Are Hinge Centers of Collective Motions. By using principal component analysis (PCA) of the atomistic trajectories, we quantified the pattern of collective motions in the SARS-CoV-2 S-RBD complexes (Figure 3). The low-frequency modes are often functionally important as perturbations induced by mutations or binding can evolve along the pre-existing slow modes to elicit specific protein responses. As may be expected given a similar topological arrangement of the SARS-CoV-2 S protein fold, the essential profiles displayed a similar shape in the complexes with nanobodies (Figure 3). The distributions of S-RBD complexes with $\mathrm{Nb20}$, VHHE, and VHHE/VHH/U nanobodies (Figure $3 \mathrm{~A}-\mathrm{C}$ ) revealed that sites of circulating variants K417 and N501 are often aligned with the local maxima corresponding to moving regions, while the L452 position belongs to the hinge region that is mostly immobilized in cooperative motions. A common sharp bifurcated peak was observed at the flexible region (residues 475-486), while the periphery of the nanobody-binding epitopes particularly E484 position is aligned with the local minimum in this region. A comparison of slow-mode profiles for the S-RBD complexes with nanobodies revealed several well-defined hinge regions, including hinge clusters that are anchored by G447/Y449 residues and also L452 and L455 positions (Figure 3B-D). Another noticeable hinge cluster includes residues F490, L492, S494, G496, N501, and Y505. Both residue clusters are located in the relatively flexible regions of the S-RBD but become largely immobilized in the complexes.

Interestingly, most of the sites of escaping mutations belong to the hinge clusters and may be involved in control of collective motions in the complexes. Indeed, the escape mutations in the VHH E interface featured G447S, Y449H/ $\mathrm{D} / \mathrm{N}, \mathrm{L} 452 \mathrm{R}, \mathrm{F} 490 \mathrm{~S}, \mathrm{~S} 494 \mathrm{P} / \mathrm{S}, \mathrm{G} 496 \mathrm{~S}$, and $\mathrm{Y} 508 \mathrm{H}$ modifications. ${ }^{61}$ We also observed that residues in the cryptic binding epitope Y369, S371, F374, and F377 are aligned with conserved hinge positions. Our analysis suggested that these hinge clusters from distant binding epitopes may cooperatively regulate the functional dynamics of the SARS-CoV-2 S-RBD complexes with nanobodies. Instructively, the residues in the moving region near E484/F486 RBD positions were aligned with the local maxima of the essential mobility profiles in the complexes with VHH E/VHH U (Figure 3C) and VHH VE 

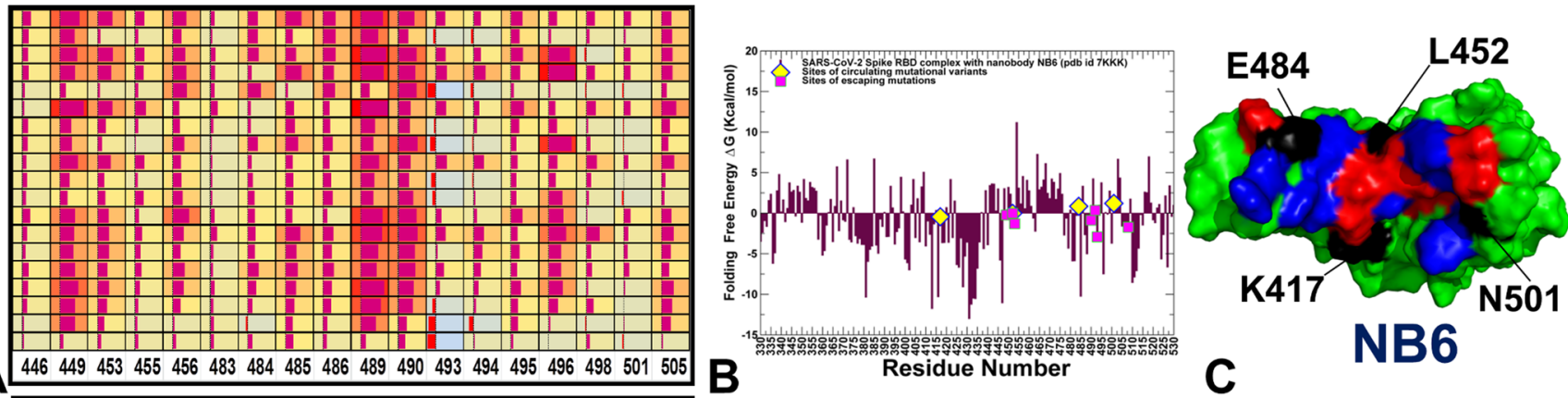

\section{B}
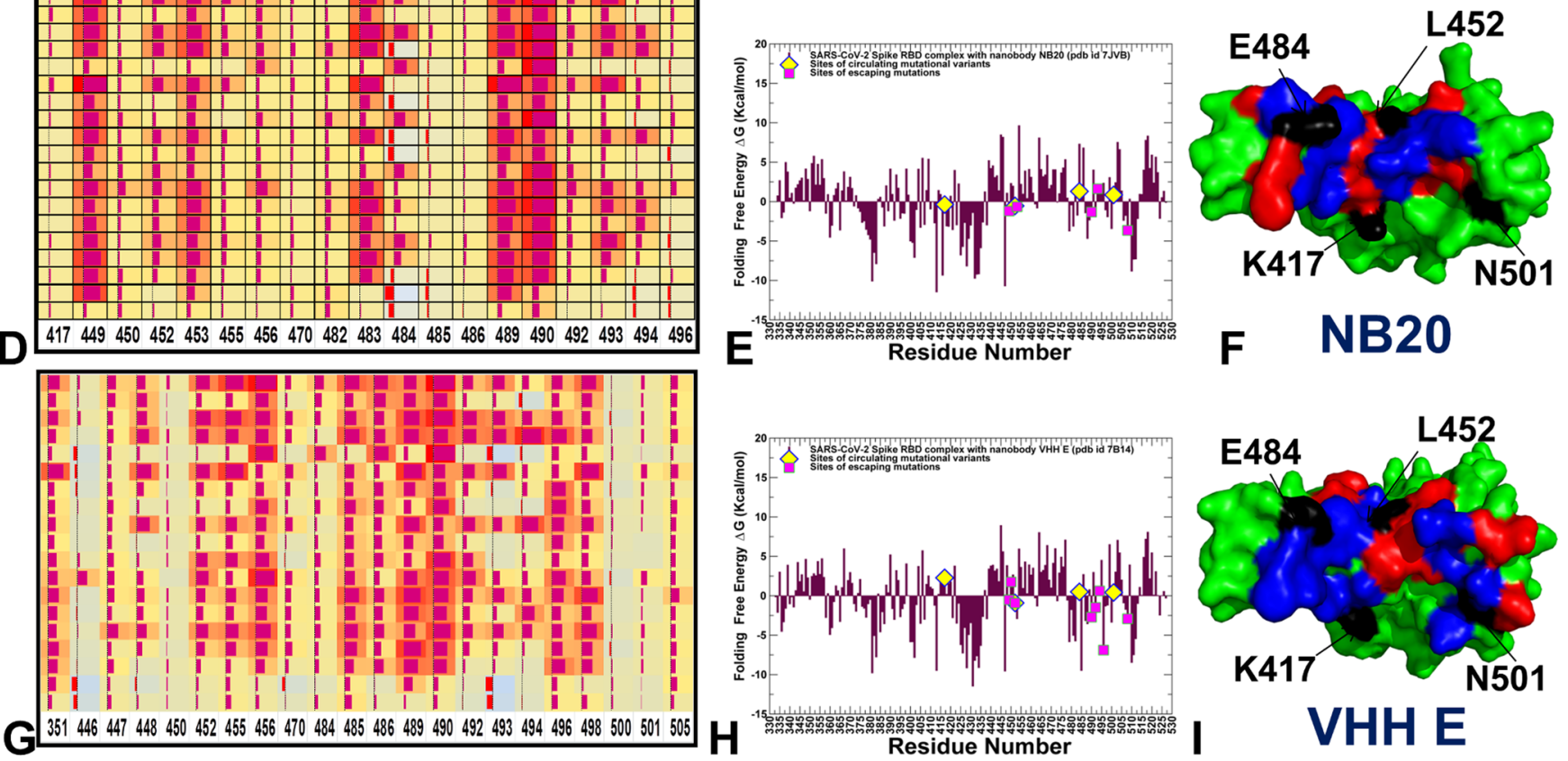

Figure 4. Mutational profiling of the SARS-CoV-2 S binding with nanobodies. (A) Mutational scanning heatmap for the SARS-CoV-2 S complex with the Nb6 nanobody. (B) Residue-based folding stability analysis of the SARS-CoV-2 S-RBD complex with Nb6. (C) Structural map of the SRBD-binding epitope in the complex with Nb6. The S-RBD is shown in the green surface. The epitope residues are shown in red and the binding energy hotspots are shown in the blue surface. The positions of K417, L452, E484, and N501 are shown in black color and annotated. (D) Mutational scanning heatmap for the SARS-CoV-2 S complex with the Nb20 nanobody. (E) Residue-based folding stability analysis of the S-RBD complex with Nb20. (F) Structural map of the S-RBD-binding epitope in the complex with $\mathrm{Nb20}$. The annotations for the epitope residues and the binding energy hotspots are shown in C. (G) Mutational scanning heatmap for the SARS-CoV-2 S complex with the VHH E nanobody, PDB id 7B14. (H) Residue-based folding stability analysis of the S-RBD complex with the VHH E nanobody. (I) Structural map of the S-RBD-binding epitope in the complex with the VHH E nanobody. The heatmaps show the computed binding free energy changes for 19 single mutations on the binding epitope sites. The squares on the heatmap are colored using a three-colored scale-from light blue to red, with red indicating the largest unfavorable effect on binding. The data bars correspond to the binding free energy changes, with the positive value shown by bars toward the right end of the cell and negative favorable values as bars oriented toward the left end. The standard errors of the mean for binding free energy changes were based on five independent trajectories, and different numbers of selected samples from a given trajectory (500, 1000, and 2000 samples) are $\sim 0.2-0.25 \mathrm{kcal} / \mathrm{mol}$ using averages over different trajectories and $\leq 0.15 \mathrm{kcal} / \mathrm{mol}$ from computations based on different numbers of samples from a single trajectory.

biparatopic nanobodies (Figure 3D). This flexible region that harbors important sites of escape mutations is involved in the propagation of collective dynamic motions. As a result, mutations in these positions may not only have effect on local dynamic changes but also alter cooperative conformational changes in the $S$ protein and compromise the fidelity of protein response to nanobody binding.

The slow-mode profiles for the S-RBD complexes with $\mathrm{CC} 12.3 / \mathrm{VHH} \mathrm{V}$ and $\mathrm{CC} 12.3 / \mathrm{VHH} \mathrm{W}$ pairs showed a distinct pattern (Figure 3E,F). The shallow minima region in the RBM-binding epitope harbored E484 and N501 positions aligned in a largely immobilized manner in collective movement regions. We also noticed that the experimentally known sites of escaping mutations for CC12.3 antibodies ${ }^{60}$ such as Y421, L455, F456, and Y508 corresponded to the local minima of the profile. According to the observed profile of collective motions, the emergence of shallow local minima regions may reflect some degrees of tolerance to mutations at individual positions as these changes would not significantly affect the pattern of functional dynamics.

The central finding of this analysis is that escape mutations can arise in the clusters of stable hinge sites and in the flexible RBM positions that belong to the moving regions along slow modes. Collectively, these residues are involved in coordination and execution of functional spike movements and dynamic control of binding. Local perturbations in structurally stable anchor positions can result in cascading dynamic changes and produce maximum response in the functionally active sites. At 

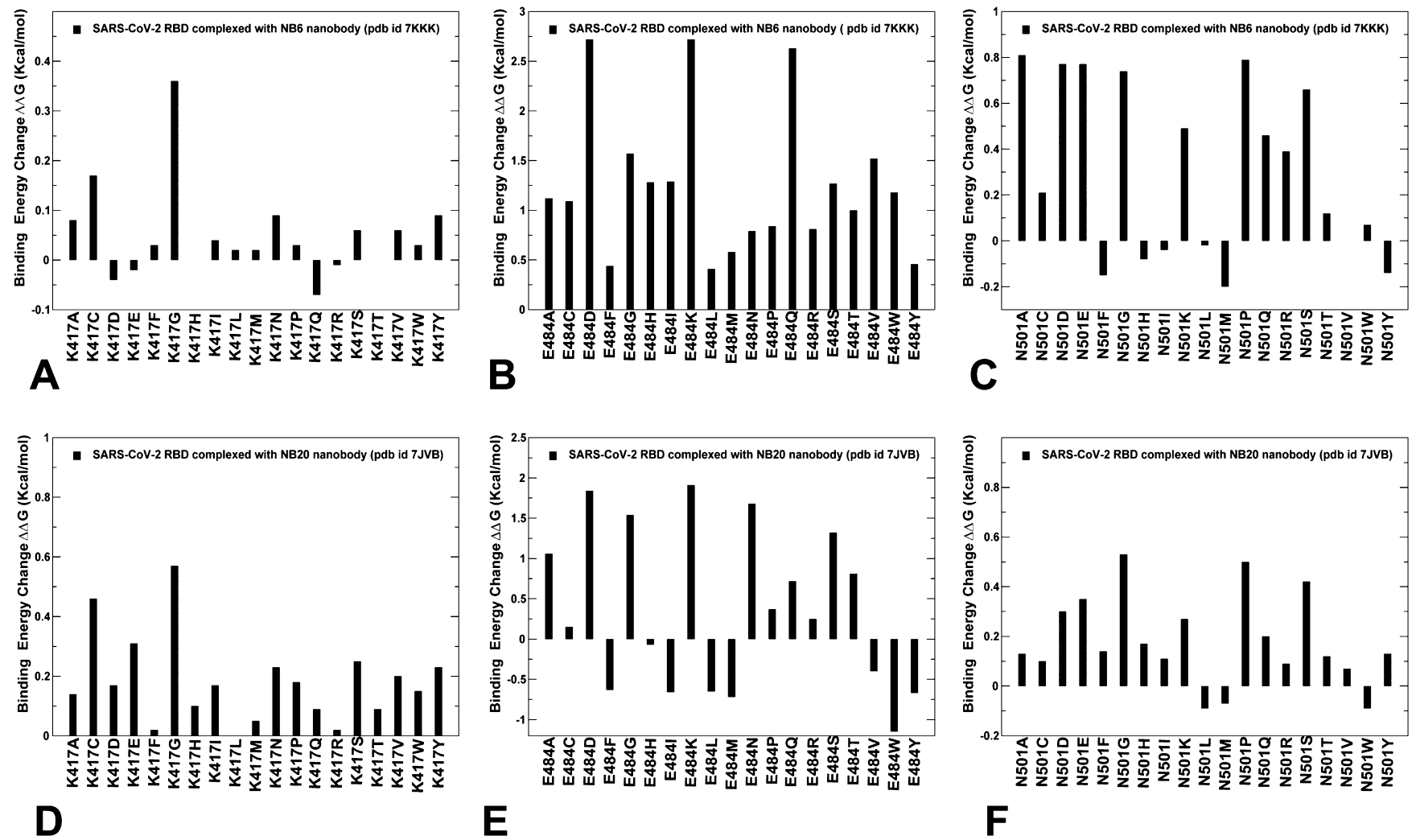

Figure 5. Mutational sensitivity analysis for the SARS-CoV-2 S complexes with $\mathrm{Nb} 6$ and $\mathrm{Nb} 20$ nanobodies. (A-C) Distribution of binding free energy changes caused by mutations of K417, E484, and N501 sites in the S-RBD complexes with the Nb6 nanobody. (D-F) Distribution of binding free energy changes caused by mutations of K417, E484, and N501 sites in the S-RBD complexes with the Nb20 nanobody. The computed standard errors of the mean for the binding free energy changes based on different numbers of selected samples from a given trajectory (500, 1000, and 2000 samples) are $0.1-0.2 \mathrm{kcal} / \mathrm{mol}$.

the same time, dynamic perturbations caused by escape mutations in residues from globally moving regions can initiate fast response along slow normal modes and trigger collective allosteric changes due to cooperativity. Combined with the conformational dynamic analysis, the results suggested that dynamic couplings between the clusters of residues targeted by escaping mutants could determine functional protein response and dictate a mechanism of the nanobody binding.

Mutational Scanning Identifies Binding Affinity Hotspots and Clarifies Energetic Drivers of NanobodyEscaping Mutations. We explored in silico mutational scanning based on the equilibrium ensembles of conformations to systematically profile the S-RBD residues and compute the binding free energy changes caused by amino acid modifications for studied SARS-CoV-2 S-RBD complexes with nanobodies. The mutational heatmaps provided a visual representation of screening, pointing to the $\mathrm{S}-\mathrm{RBD}$ residues with high sensitivity to modifications and therefore considered as potential binding energy hotspots. We also used the FoldX approach with the all-atom representation of the protein structure $^{87-90}$ to simultaneously evaluate the folding free energies for the S-RBD residues and identify hotspots of protein stability in the complexes. The protein stability $\Delta \Delta G$ changes were computed by averaging the results over 1000 trajectory samples. ${ }^{91,92}$

Mutational analysis of the S-RBD binding with Nb6 revealed binding energy hotspots for the group of hydrophobic residues (Y449, L453, L455, F456, G485, Y489, F490, G496, and Y505) that were experimentally confirmed as important residues for ACE2 binding in deep mutagenesis scanning (Figure 4A,B). ${ }^{39}$ Our results indicated that a strong similarity in the interaction pattern with ACE2 may provide the energetic advantage for the $\mathrm{Nb} 6$ nanobody to competitively inhibit the ACE-binding region. The folding free energies of the $S$-RBD residues accurately reproduced stability of the conserved RBD core (Figure 4B). The negative folding free energies were seen for the binding energy hotspots Y449, L455, F456, G485, Y489, and Y505, suggesting that these hydrophobic sites play an important role in both protein stability and binding with the nanobody. This may explain why escape mutations produced by virus tend to "avoid" these positions that are central for the RBD integrity and productive binding to the host receptor. Structural map of the binding epitope residues showed that K417, L452, E484, and N501 are located in the immediate proximity of the binding hotspot residues (Figure 4C). Interestingly, mutational scanning also reproduced sensitivity of sites targeted by common resistant mutations of many individual nanobodies (F490S, E484K, Q493K, F490L, F486S, F486L, and Y508H). ${ }^{58}$ In particular, E484, F486, and F490 positions appeared to be highly sensitive to $\mathrm{Nb} 6$ binding (Figure $4 \mathrm{~A}-\mathrm{C}$ ). The binding energy hotspots in the complex with the $\mathrm{Nb} 20$ nanobody corresponded to residues Y449, Y453, V483, Y489, F490, and Q493 (Figure 4D). We found a number of commonly shared hotspots for $\mathrm{Nb} 6$ and $\mathrm{Nb} 20$ nanobodies including Y449, F456, Y489, and F490 (Figure 4D). The nanobody-specific binding hotspots for $\mathrm{Nb} 20$ were aligned with V483, L492, and Q493 residues (Figure 4D). 

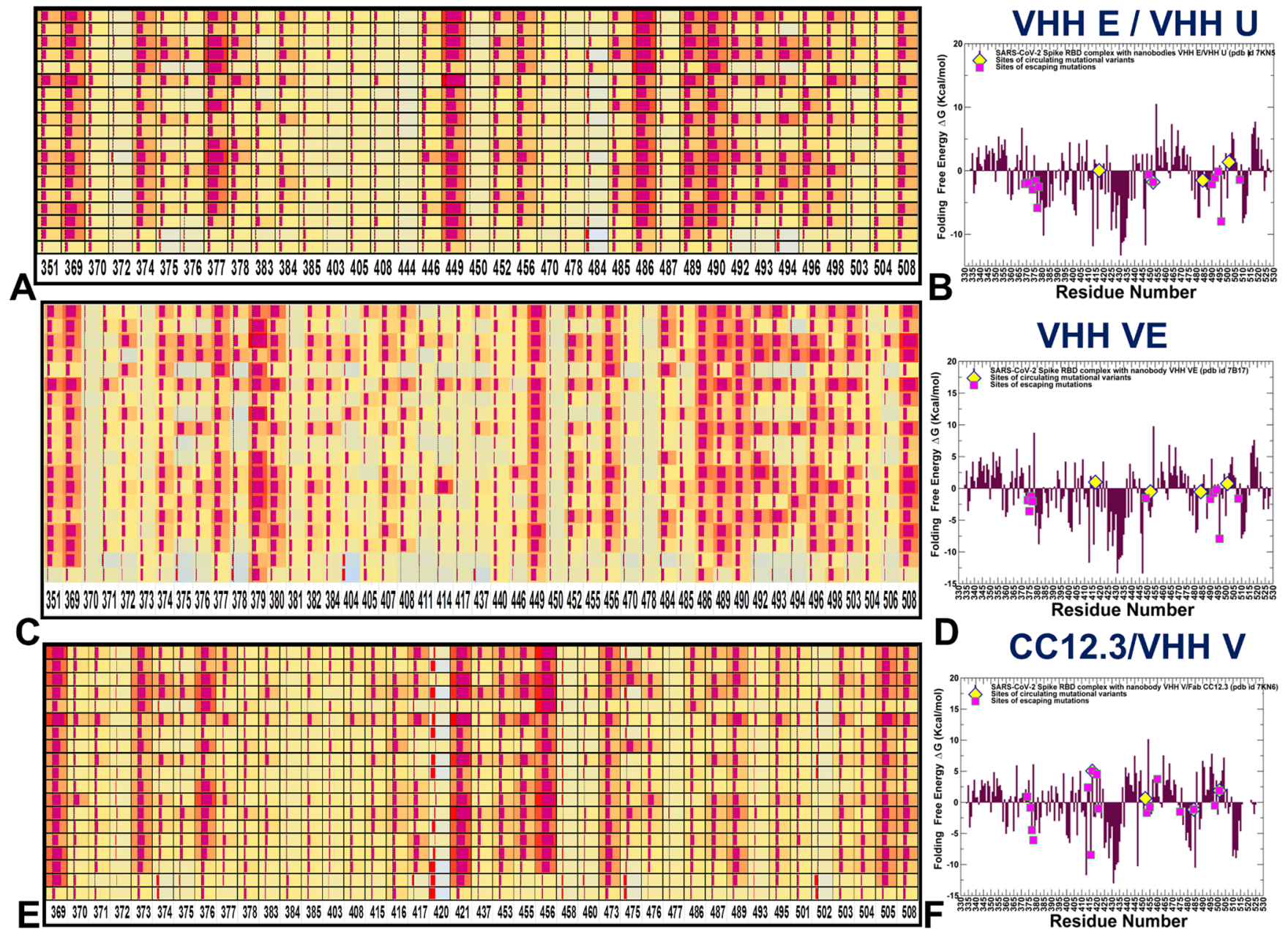

Figure 6. Mutational scanning heatmap for the SARS-CoV-2 S complex with VHHE/VHH U combination of nanobodies, PDB id 7KN5 (A). Folding stability analysis of the S-RBD complex with VHH E/VHH U combination (B). Mutational scanning heatmap for the S-RBD complex with the VHH VE biparatopic nanobody, PDB id 7B17 (C). Residue-based folding stability analysis of the S-RBD complex with VHH VE (D). Mutational scanning heatmap for the SARS-CoV-2 S complex with CC12.3/VHH V combination, PDB id 7KN6 (E). Folding stability analysis of the S-RBD complex with CC12.3/VHH V antibody/nanobody combination (F). The heatmaps show the computed binding free energy changes for 19 single mutations on the binding epitope sites. The squares on the heatmap are colored using a three-colored scale-from light blue to red, with red indicating the largest unfavorable binding changes.

In agreement with the experimental studies, mutational scanning analysis showed that F490, E484, F486, and Q493 positions can be important sites of escaping mutations for $\mathrm{Nb6}$ and $\mathrm{Nb20}$ nanobodies (Figure 4A,D). Several clusters of binding energy hotspots were found for the S-RBD complex with the VHH E nanobody (Figure 4G-I). The first included sites L452, Y453, and F456, while the second cluster included functional positions G485, F486, Y489, F490, L492, G496, and Q498. We specifically focused on the mutational profiling of K417, E484, and N501 sites targeted by global circulating mutations (Figure 5). Amino acid substitutions in K417 and $\mathrm{N} 501$ positions produced relatively small changes in $\mathrm{Nb6}$ binding, while most of the mutations at E484 yielded strong unfavorable effects on binding affinity (Figure $5 \mathrm{~A}-\mathrm{C}$ ). Notably, mutations of the E484 residue resulted in a significant loss of binding affinity $(>2.0 \mathrm{kcal} / \mathrm{moll})$ for both $\mathrm{Nb} 6$ and $\mathrm{Nb} 20$ nanobodies, while the respective modifications are known to improve binding with the host receptor (Figure 5). In the complex with $\mathrm{Nb} 20, \mathrm{E} 484 \mathrm{D} / \mathrm{K} / \mathrm{Q}$ mutations appeared to be highly unfavorable for binding, resulting in $>2.5 \mathrm{kcal} / \mathrm{mol}$ losses in the binding affinity (Figure 5E). Consistent with the results of mutational scanning, the engineered charge reversal on R31 (R31D) is unable to recover the salt bridge and restore binding to the E484K mutant. ${ }^{56,57}$ At the same time, the N501Y mutation led to the moderately increased binding affinity (Figure 5F). Structural mapping of protein stability hotspots for $\mathrm{Nb} 6$ and $\mathrm{Nb} 20$ nanobodies highlighted distinct conserved clusters of residues localized at the center of the RBM-binding epitope and in the S-RBD core region near the cryptic binding epitope (Supporting Information, Figure S5A,B). Collectively, these observations are in agreement with the experimental studies showing that E484 on the RBD is the "Achilles heel" of the ultrapotent $\mathrm{Nb} 20$ and $\mathrm{Nb} 21$ nanobodies. ${ }^{56,57}$ These results strongly support the notion that functional and structural plasticity can be important to mechanisms by which mutations can alter the antigenic responses and elicit antibody evasion. Indeed, we found that the sites of mutational escape to nanobodies corresponded to flexible energetic centers that are involved in the network of local binding interactions and execution of functional cooperative movements in response to binding. As a result, mutations in these positions could have strong cumulative effects by compromising nanobody recognition and binding affinity as well as affecting functional dynamics and protein 


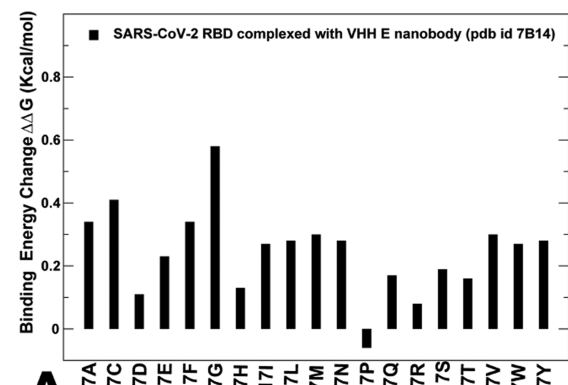

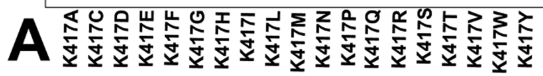
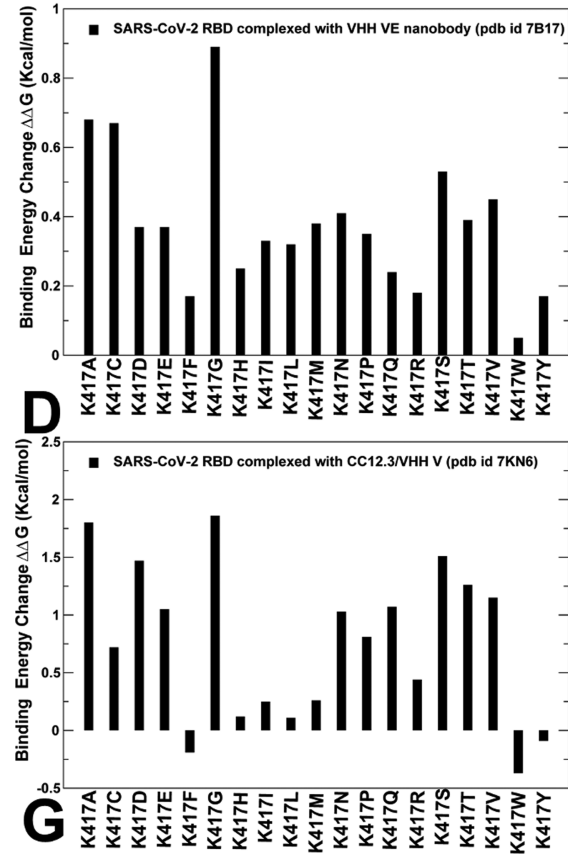
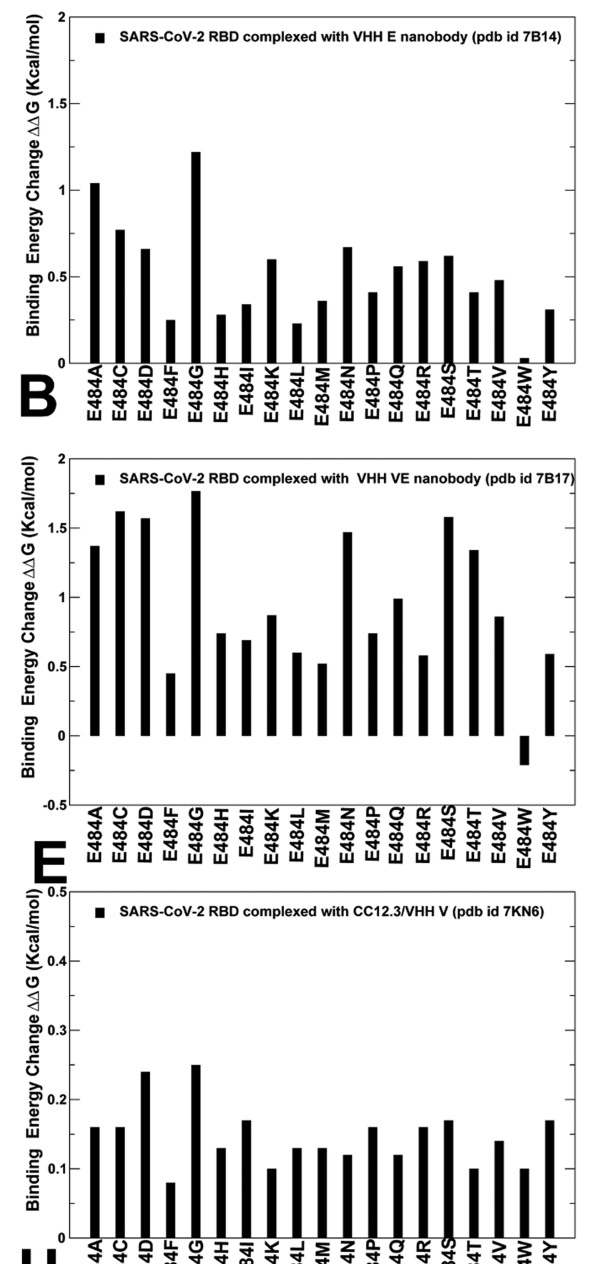

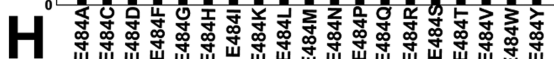
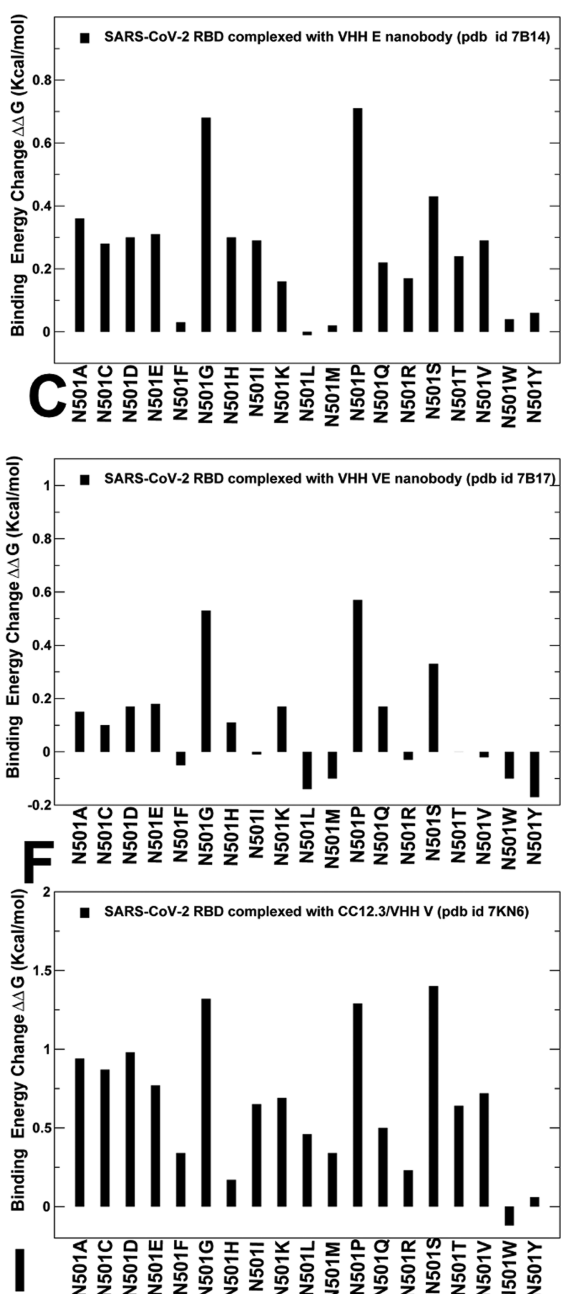

Figure 7. Mutational sensitivity analysis for the SARS-CoV-2 S complexes with VHH E, VHH VE nanobodies, and CC12.3/VHH V antibody/ nanobody combination. (A-C) Distribution of binding free energy changes caused by mutations of K417, E484, and N501 sites in the S-RBD complexes with the VHH E nanobody. (D-F) Distribution of binding free energy changes caused by mutations of K417, E484, and N501 sites in the S-RBD complexes with the VHH VE biparatopic nanobody. (G-I) Distribution of binding free energy changes caused by mutations of K417, E484, and N501 sites in the S-RBD complexes with CC12.3/VHH V antibody/nanobody combination. The standard errors of the mean for the binding free energy changes are $0.15-0.2 \mathrm{kcal} / \mathrm{mol}$.

response to perturbations. These results support the recent study suggesting that functional plasticity is instrumental to allosteric communications in proteins where structural stability centers cooperate with more dynamic allosteric hotspots that correspond to structurally adaptable and moderately conserved protein positions. $^{93}$

Binding Footprints for Nanobody Combinations at Distinct Epitopes Reveal Changes in the Mutational Landscape and Increased Resilience to Escape Mutations. We also identified binding affinity fingerprints for the SARS-CoV-2 S-RBD complexes with a nanobody combination $\mathrm{VHH}$ E/VHH U targeting different epitopes (Figure 6A,B) and the biparatopic nanobody $\mathrm{VHH}$ VE that has two antigenbinding sites in one molecule and obtained by fusing nanobodies that targeted distinct epitope regions (Figure 6C,D). For the S-RBD complex with the nanobody pair VHHE/VHH U, the number of RBD residues involved in the favorable binding interactions increased, but the number of binding energy hotspots remained similar to that of the complexes with single nanobodies (Figure 5A). The S-RBD hotspot residues were aligned with the same group of residues Y449, F486, Y489, F490, and Y508 (Figure 5A). In agreement with the experiments, ${ }^{61}$ mutations at the VHH E interface $\mathrm{Y} 449 \mathrm{H} / \mathrm{D} / \mathrm{N}, \mathrm{F} 490 \mathrm{~S}, \mathrm{~S} 494 \mathrm{P} / \mathrm{S}, \mathrm{G} 496 \mathrm{~S}$, and $\mathrm{Y} 508 \mathrm{H}$ produced large unfavorable $\Delta \Delta G$ changes $>2.0 \mathrm{kcal} / \mathrm{mol}$ (Figure 6A,B). A number of hotspot positions were also observed in the second cryptic epitope including conserved and stable residues Y369, S371, F374, and F377.

Mutational scanning analysis also showed that escaping mutations Y369H, S371P, F374I/V, T376I, F377L, and $\mathrm{K} 378 \mathrm{Q} / \mathrm{N}$ at the $\mathrm{VHH} \mathrm{U}$ interface resulted in considerable binding losses with $\Delta \Delta G \sim 2.5 \mathrm{kcal} / \mathrm{mol} /$ (Figure 6A,B). In the complex with the biparatopic nanobody VHH VE (Figure $6 \mathrm{C}, \mathrm{D})$, the pattern remained generally similar, pointing to a strong contribution of residues F377, C379, Y449, F486, Y489, L490, and L492. The binding interactions in the cryptic epitope are determined by RBD core residues 369-384. Of importance are strong contributions of F377, C379, and Y380 residues acting as rigid binding energy hotspots (Figure 6C).

Many of the binding hotspot centers for complexes with the $\mathrm{VHH} \mathrm{E} / \mathrm{VHH} \mathrm{U}$ pair and $\mathrm{VHH} \mathrm{VE}$ corresponded to conserved centers of protein stability (Figure 6A-D) where mutations could affect the RBD folding stability, partly explaining why these nanobodies can suppress the emergence 
of escaping variants. Even though these nanobodies make contacts with sites of circulating variants K417, L452, and E484, these positions can be quite tolerant to mutations (Figure 6C,D). This is consistent with the observed effectiveness of the nanobodies against many antigenic variants. $^{61}$ The results also indicated that only moderately flexible RBD sites F486 and F490 are consistently featured as common binding energy hotspots for these complexes, which may explain why escape mutants in these positions are known to dominate at the $\mathrm{VHH}$ E interface. ${ }^{61}$ The important finding of this analysis is that mutational sensitivity of the binding hotspots in the RBM epitope can be attenuated in the complex with the biparatopic nanobody. This may contribute to the increased resilience of the $\mathrm{VHH} \mathrm{VE}$ engineered nanobody to mutational escape. The mutational heatmaps of the S-RBD binding with antibody CC12.3 combinations with VHH V and $\mathrm{VHH} W$ nanobodies (Figure 6E,F) recapitulated the experimentally known sites of escaping mutations Y421, L455, F456, and Y508 for CC12.3. ${ }^{60}$

The computed standard errors of the mean for the binding free energy changes were $\sim 0.13-0.22 \mathrm{kcal} / \mathrm{mol}$ using averages over different trajectories and within $0.1 \mathrm{kcal} / \mathrm{mol}$ from computations based on different numbers of samples from a single trajectory. A more detailed analysis of mutational changes in sites of circulating variants showed that VHH E and $\mathrm{VHH}$ VE nanobodies retained their binding affinity to mutations in K417 and N501 positions and remained relatively forgiving to E484 mutations (Figure $7 \mathrm{~A}-\mathrm{F}$ ). Our analysis correctly captured a considerably better tolerance of these nanobodies to E484K/D mutations that are sensitive to the ultrapotent $\mathrm{Nb} 20$ and $\mathrm{Nb} 21$ class I nanobodies (Figure 5). ${ }^{56,57}$

According to our analysis, binding of $\mathrm{CC} 12.3 / \mathrm{VHH} \mathrm{V}$ and $\mathrm{CC} 12.3 / \mathrm{VHH} \mathrm{W}$ combinations can result in even stronger tolerance to mutations as only small binding energy differences induced by mutations were found for these complexes (Figure $7 \mathrm{G}-\mathrm{I}$ ). This is consistent with the observed resilience of these cocktails against these antigenic variants. ${ }^{61}$

Structural maps of the RBM-binding footprints showed that sites of circulating variants are located at the edges of the binding hotspot regions, and therefore, mutations in these positions appeared to have a relatively moderate effect on binding (Figure 8). Of importance are only minor overlaps of the critical E484 site with the regions involved in binding with VHH E and VHH VE nanobodies (Figure 8D,E). Even more instructive are the structural maps for $\mathrm{CC} 12.3 / \mathrm{VHH} \mathrm{V}$ antibody/nanobody combination (Figure $8 \mathrm{C}, \mathrm{F}$ ). In this case, the E484 site has no direct overlap with the binding hotspot regions, while other positions K417 and N501 are only peripherally involved in the interaction footprint. The large binding epitope and broadly distributed binding hotspot positions can enable high affinity while inducing the increased resilience of these synergistic cocktails to common escape mutants. Structural mapping showed a significant coverage of the binding energy hotspots at the second binding epitope. Our results suggest that binding to stable RBD core residues in this site can be leveraged by the biparatopic nanobody and antibody/nanobody combination as a regulatory anchor that firmly immobilizes nanobodies at the cryptic binding site and facilitates binding of the VHH E arm at the RBM epitope at a smaller entropic cost. This suggests a plausible mechanism by which biparatopic nanobodies can leverage dynamic couplings between distinct binding epitopes to enhance binding and mitigate mutational escape.
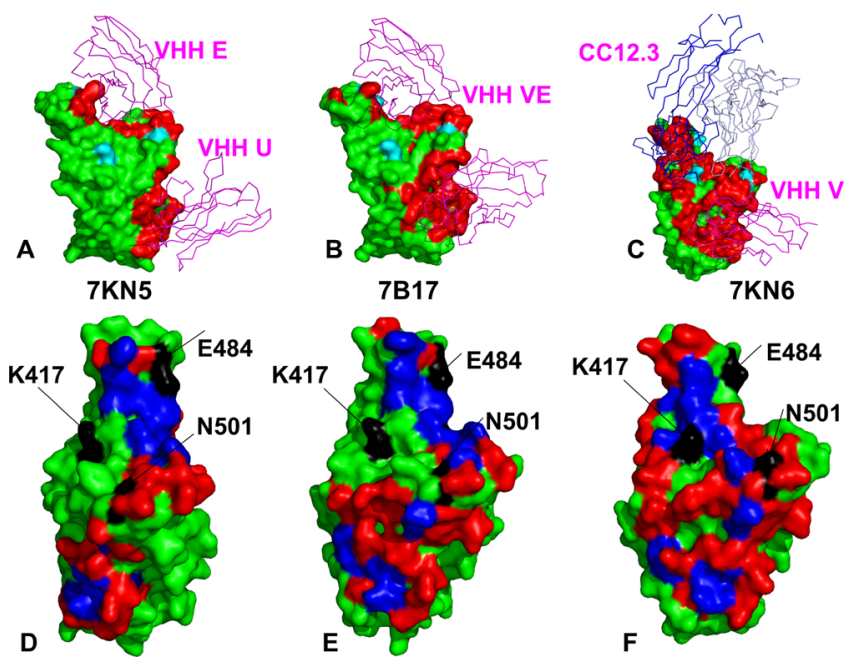

Figure 8. Structure of the S-RBD complex with VHH E/VHH U (A), complex with the biparatopic nanobody VHH VE (B), and CC12.3/ VHH V antibody/nanobody combination (C). The S-RBD is shown in the green surface. The epitope residues are shown in red. The positions of K417, L452, E484, and N501 are in light cyan color. Top views of the binding epitopes for S-RBD complexes with VHH E/ VHH U (D), complex with biparatopic nanobody VHH VE (E), and CC12.3/VHH V pair (F). The S-RBD is shown in the green surface. The epitope residues are shown in red and the binding energy hotspots are shown in the blue surface. The positions of K417, L452, E484, and N501 are shown in black color and annotated.

We also evaluated the correlations between binding free energy changes of the S-RBD residues with ACE2 and studied nanobodies (Figure 9). A consistent correlation (Pearson correlation coefficient $R \sim 0.7-0.85$ ) was found between the effects of RBD mutations on binding with ACE2 and the nanobodies. This indicated that the nanobodies can efficiently mimic the binding energetics of the host receptor. We specifically highlighted the binding free energy changes associated with mutations at sites of circulating variants K417, L452, E484, and N501 (Figure 9). While mutations in these positions tend to cause similar and moderate binding energy changes for ACE2 and nanobodies, there was some dispersion of energetic changes showing a larger unfavorable effect on nanobody binding $(\sim 1.0-1.5 \mathrm{kcal} / \mathrm{mol})$ than on ACE2 binding $(\sim 0.5 \mathrm{kcal} / \mathrm{mol})$ (Figure $9 \mathrm{~A}-\mathrm{C})$. A very strong correlation was seen between binding free energy changes in these functional positions with ACE2 and CC12.3/VHH V combination (Figure 9D). This may reflect a high degree of tolerance to mutations in sites of circulating variants, suggesting that a combination of antibody and nanobody binding may be very efficient in the suppression of mutational escape. Hence, the results suggest that structural and dynamic mimicry of the protein stability and binding interactions by the nanobody combinations could limit the escaping potential for the virus in sites of common circulating variants.

In Silico Profiling of the SARS-CoV-2 Binding with Nanobodies and Predicted Mutational Escape Landscape: A Comparison with Mutagenesis Experiments. The results of our study provided a quantitative characterization of the SARS-CoV-2 S binding with a panel of nanobodies that agrees with a wide spectrum of mutagenesis and biophysical experiments. Here, we summarize our findings and their connection with the available experimental data. From the structural and dynamic analysis of SARS-CoV-2 S 

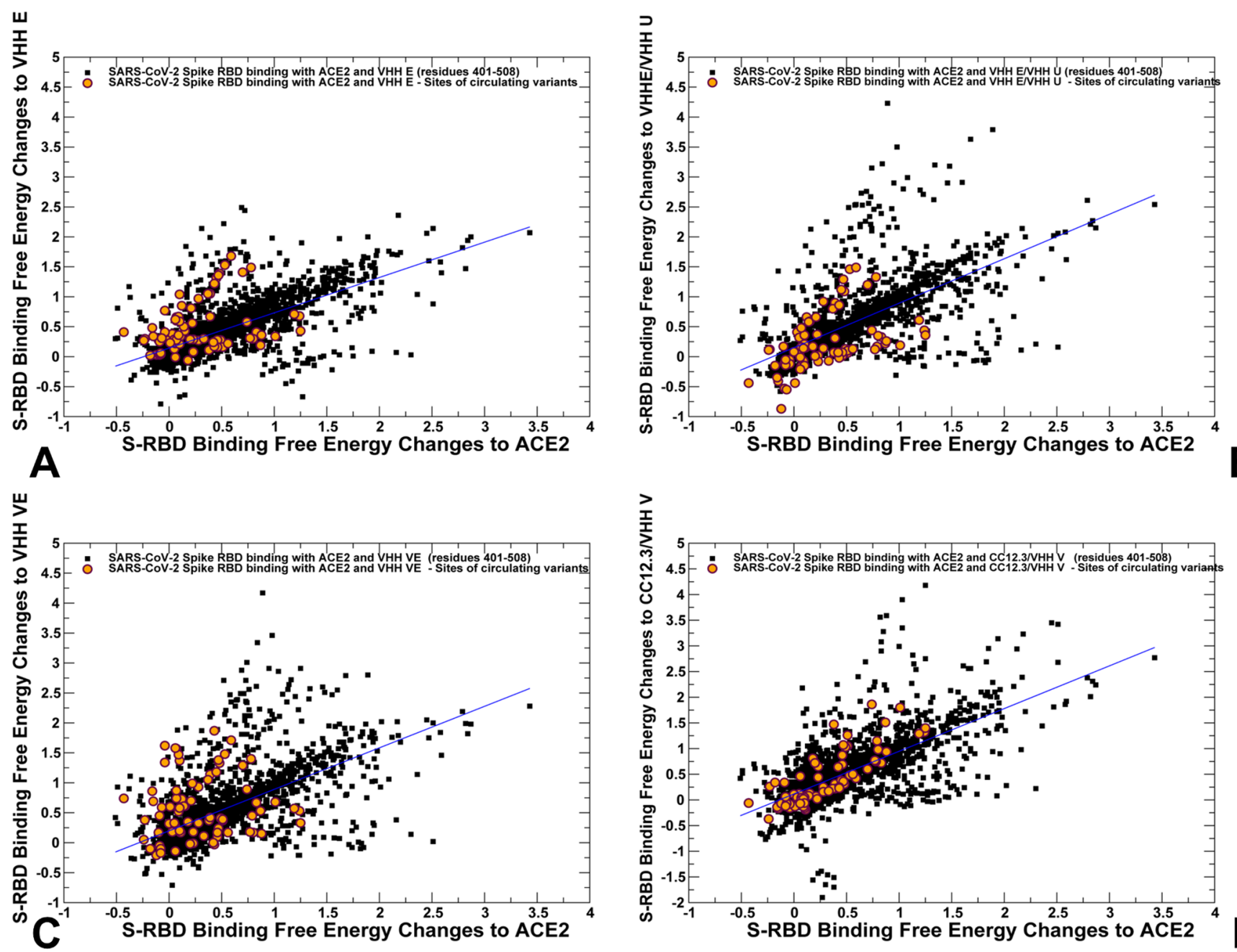

Figure 9. Scatter plots of binding free energy changes for the S-RBD residues in complexes with ACE2 and VHH E (A), ACE2 and VHH E/VHH $\mathrm{U}(\mathrm{B}), \mathrm{ACE} 2$ and VHH VE (C), and ACE2 and CC12.3/VHH V combination (D). The data points are shown as black-filled squares and scatter points corresponding to the binding free energy changes in sites K417, L452, E484, and N501 are shown as orange-filled circles.

binding with the $\mathrm{Nb} 6$ nanobody, we found that $\mathrm{Nb} 6$ binding is highly sensitive to modifications at the E484 site, particularly E484K mutation leading to a significant loss of binding affinity. Indeed, the experiments revealed that binding of the highly potent $\mathrm{Nb} 6$ nanobody can be severely compromised by E484K mutation, resulting in the loss of activity. ${ }^{55}$ The computed mutational profiling maps indicated that the experimentally known resistant mutations F490S, E484K, Q493K, F490L, F486S, and F486L are indeed highly sensitive to Nb6 binding. Hence, in silico mutational profiling reproduced the known binding energy hotspots responsible for the high potency of $\mathrm{Nb6}$ and provided accurate predictions of nanobody-sensitive positions and escape mutants. We also found that E484D/K/Q mutations were highly unfavorable for $\mathrm{Nb} 20$ binding, while $\mathrm{L} 452 \mathrm{R}$ and $\mathrm{L} 452 \mathrm{~K}$ variants were less deleterious than the modifications in E484. These results are in good agreement with the mutagenesis studies of $\mathrm{Nb} 20$ binding, ${ }^{56}$ indicating that amino acid substitutions in E484 and L452 sites can be detrimental and allow virus by targeting these vulnerable sites to elicit a strong escape from the nanobody.

In agreement with the experimental studies, in silico mutational profiling accurately predicted F490, E484, F486, and Q493 positions as common sites of escaping mutations for both $\mathrm{Nb6}$ and $\mathrm{Nb} 20$ nanobodies. Our results provided a plausible rationale to the functional role of E484 as the "Achilles heel" of the ultrapotent nanobodies as a range of modifications in this site can dramatically reduce the affinity of these class I nanobodies. ${ }^{55-57}$ Consistent with the functional studies, we found that the binding of nanobodies $\mathrm{Nb} 6, \mathrm{Nb} 20$, and $\mathrm{VHH}$ E can be impeded by perturbations at highly antigenic sites in the RBM region (particularly E84 position) as escape mutations tend to target these flexible positions because of their effect on collective dynamic changes and allosteric interactions. Of special interest is also a strong agreement between computed mutational scanning profiles and the experimentally observed pattern of escape mutations in complexes with $\mathrm{VHH}$ E, VHH E/VHH U, and VHH VE nanobodies. Consistent with the mutagenesis screens, ${ }^{61}$ our results identified prominent escape mutations $\mathrm{Y} 449 \mathrm{H} / \mathrm{D} / \mathrm{N}$, L452R, F490S, S494P, and Y508H in the VHH E interface, as well as a group of escape mutations targeting in the conserved sites of the RBD core (S371, F374, T3765, and F377) at the VHH U interface.

In silico mutational scanning and the computed binding free energy changes using the BeAtMuSiC approach ${ }^{94,95}$ showed a good overall agreement with mutagenesis experiments, indicating that the employed computational tools may be particularly useful for the detection of hotspots of escape 
mutations and identification of major mutations that are deleterious for nanobody binding. The advantages of this approach are fast and accurate predictions of the effect of mutations on both the strength of the binding interactions and on the stability of the complex using statistical potentials and neural networks. This approach is comparable to other knowledge-based structural methods such as Dcomplex ${ }^{96}$ which is based on distance-specific contact potentials trained on the monomer structures. By combining structural and evolutionary profile analyses derived from the binding interfaces, the BindProfX approach ${ }^{97}$ showed potential for improvements over both the statistics-based and physics-based FoldX potentials. ${ }^{90-92}$ The BeAtMuSiC approach adapted in our study that was further enhanced through ensemble-based averaging of binding energy computations yielded a good correlation between prediction and experiments and was successfully used for the binding free analysis of the SARSCoV-2 S binding with the host receptor ${ }^{77}$ and antibodies. ${ }^{80-82}$ The recent analysis of computational tools for estimating protein-protein binding probed several approaches or their predictive ability of relative binding affinities for 654 single mutations on antibody-antigen and nonantibody-antigen complexes. $^{98}$ Instructively, this analysis showed contextdependent and system-dependent strengths and weaknesses of these tools. Moreover, physics-based methods often display a similar predictive power to comparable but much faster knowledge-based methods which are indispensable for massive mutational scanning experiments. ${ }^{98}$ Importantly, it was concluded that most methods can quickly and accurately differentiate between favorable and unfavorable mutations rather than accurately predicting the actual binding affinities. ${ }^{98}$

Our results concur with these observations showing that the rapid in silico profiling of mutations for the SARS-CoV-2 S protein complexes can yield a fairly robust assessment of major variants and suggest structural basis for changes in the protein stability and binding. Interestingly, a recent large-scale in silico mutagenesis study using the BeAtMuSiC approach profiled all possible point mutations in the RBD residues on its stability and binding with antibodies and ACE2 receptors, showing that predictions agreed well with various experimental, epidemiological, and clinical data. ${ }^{99}$ It is worth stressing that more rigorous physics-based approaches may yield more accurate predictions of binding affinity, but the requirements for adequate sampling and comprehensive scanning of protein residues make this strategy less plausible in practice. Instead, our approach by combining atomistic simulations and knowledge-based models of protein binding provided the ensemble-average estimates of the binding free energy changes allowing for a comprehensive scanning and meaningful interpretation of the SARS-CoV-2 S binding with nanobodies.

\section{CONCLUSIONS}

We combined atomistic simulations with the ensemble-based mutational profiling of binding interactions for a diverse panel of SARS-CoV-2 S-RBD complexes with nanobodies. The analysis of collective dynamics in the nanobody complexes showed that escape mutations at the RBM epitope may emerge in the intrinsically dynamic positions that become less flexible upon binding and play an important regulatory role in coordination of functional motions. Using the in silico mutational profiling approach for probing the protein stability and binding, we examine dynamics and energetics of the SARS-CoV-2 complexes with single nanobodies $\mathrm{Nb} 6$ and
$\mathrm{Nb20}$, VHH E, a pair combination $\mathrm{VHH} \mathrm{E}+\mathrm{U}$, a biparatopic nanobody $\mathrm{VHH} \mathrm{VE}$, and a combination of the CC12.3 antibody and $\mathrm{VHH} \mathrm{V/W}$ nanobodies. This study characterizes the binding energy hotspots in the SARS-CoV-2 protein and complexes with nanobodies providing a quantitative analysis of the effects of circulating variants and escaping mutations on binding that is consistent with a broad range of biochemical experiments. The results also revealed that the biparatopic nanobody can use binding at the cryptic binding site to establish a structurally stable regulatory "anchor" that mediates dynamic couplings between nanobody arms and provides allosteric control over structural changes in the RBM epitope. This suggests a plausible mechanism by which biparatopic nanobodies can leverage dynamic couplings to synergistically inhibit distinct binding epitopes and suppress mutational escape. We argue that mutational escape mechanisms may be controlled through structurally adaptable binding hotspots in the receptor-accessible binding epitope that are dynamically coupled to the stability centers in the distant binding epitope targeted by $\mathrm{VHH} \mathrm{U} / \mathrm{V} / \mathrm{W}$ nanobodies. These results may prove to be useful to identify the emergent strains of concern, better understand, and combat the escaping mutation mechanisms which may aid in the rational design of new vaccines and nanobody/antibody combinations.

\section{METHODS}

MD Simulations. All structures were obtained from the Protein Data Bank. ${ }^{100,101}$ Hydrogen atoms and missing residues were initially added and assigned according to the WHATIF program web interface. ${ }^{102}$ The structures were further preprocessed through the Protein Preparation Wizard (Schrödinger, LLC, New York, NY). The missing loops in the cryo-EM structures were also reconstructed using templatebased loop prediction approaches ArchPRED. ${ }^{103}$ The side chain rotamers were refined and optimized by the SCWRL4 tool. ${ }^{104}$ The protein structures were subsequently optimized using atomic-level energy minimization with a composite physics and knowledge-based force fields using the 3Drefine method. ${ }^{105}$ The shielding of the receptor binding sites by glycans is an important feature of viral glycoproteins, and glycosylation on SARS-CoV-2 proteins can camouflage immunogenic protein epitopes. ${ }^{106,107}$ The structure of glycans at important glycosites of the closed and open states of SARSCoV-2 $S$ protein was previously determined in the cryo-EM structures of the SARS-CoV-2 spike $S$ trimer in the closed state (PDB id 6VXX) and open state (PDB id 6VYB). These glycans were incorporated in simulations in addition to the experimentally resolved glycan residues present in the cryoEM structures of studied SARS-CoV-2 S proteins.

All-atom $1 \mu \mathrm{s}$ MD simulations have been performed for all studied protein structures. The structures of the SARS-CoV-2 $S$ trimers were simulated in a box size of $85 \times 85 \times 85 \AA$ with a buffering distance of $12 \AA$. Assuming normal charge states of ionizable groups corresponding to $\mathrm{pH}=7$, sodium $\left(\mathrm{Na}^{+}\right)$and chloride $\left(\mathrm{Cl}^{-}\right)$counter ions were added to achieve charge neutrality, and a salt concentration of $0.15 \mathrm{M} \mathrm{NaCl}$ was maintained. All $\mathrm{Na}^{+}$and $\mathrm{Cl}^{-}$ions were placed at least $8 \AA$ away from any protein atoms and from each other. All-atom MD simulations were performed for an $\mathrm{N}, \mathrm{P}, \mathrm{T}$ ensemble in the explicit solvent using the NAMD 2.13 package ${ }^{108}$ with the CHARMM36 force field. ${ }^{109}$ The nonbonded van der Waals interactions were computed using an atom-based cutoff of 12 $\AA$ 就 with switching van der Waals potential beginning at $10 \AA$. 
Long-range electrostatic interactions were calculated using the particle mesh Ewald method. ${ }^{110}$ All atoms of the complex were first restrained at their crystal structure positions with a force constant of $10 \mathrm{Kcal} \mathrm{mol}^{-1} \AA^{-2}$. Equilibration was done in steps by gradually increasing the system temperature in steps of $20 \mathrm{~K}$ starting from $10 \mathrm{~K}$ until $310 \mathrm{~K}$ and, at each step, $1 \mathrm{~ns}$ equilibration was done maintaining a restraint of $10 \mathrm{Kcal} \mathrm{mol}^{-1}$ $\AA^{-2}$ on the protein $C_{\alpha}$ atoms. After the restraints on the protein atoms were removed, the system was equilibrated for an additional $10 \mathrm{~ns}$. An NPT production simulation was run on the equilibrated structures for $500 \mathrm{~ns}$ maintaining the temperature at $310 \mathrm{~K}$ and a constant pressure (1 atm). In simulations, the Nose-Hoover thermostat ${ }^{111}$ and isotropic Martyna-Tobias-Klein barostat ${ }^{112}$ were used to maintain the temperature at $310 \mathrm{~K}$ and pressure at 1 atm, respectively.

We performed PCA of reconstructed trajectories derived from CABS-CG simulations using the CARMA package ${ }^{113}$ and also determined the essential slow-mode profiles using elastic network model (ENM) analysis. ${ }^{114-116}$ Two ENMs: Gaussian network model (GNM) and anisotropic network model (ANM) approaches were used to compute the amplitudes of isotropic thermal motions and directionality of anisotropic motions. The functional dynamic analysis was conducted using the GNM in which the protein structure is reduced to a network of $\mathrm{N}$ residue nodes identified by $\mathrm{C}_{\alpha}$ atoms and the fluctuations of each node are assumed to be isotropic and Gaussian. Conformational mobility profiles in the essential space of low-frequency modes were obtained using the ANM server $^{116}$ and DynOmics server. ${ }^{117}$

Mutational Scanning and Sensitivity Analysis. We conducted mutational scanning analysis of the binding epitope residues for the SARS-CoV-2 S protein complexes. Each binding epitope residue was systematically mutated using all 19 substitutions, and the corresponding protein stability changes were computed. The BeAtMuSiC approach ${ }^{94,95}$ was employed that is based on statistical potentials describing the pairwise interresidue distances, backbone torsion angles, and solvent accessibilities and considers the effect of the mutation on the strength of the interactions at the interface and on the overall stability of the complex. The binding free energy of the protein-protein complex can be expressed as the difference in the folding free energy of the complex and folding free energies of the two protein binding partners

$$
\Delta G_{\text {bind }}=G^{\mathrm{com}}-G^{\mathrm{A}}-G^{\mathrm{B}}
$$

The change of the binding energy due to a mutation was then calculated as follows

$$
\Delta \Delta G_{\text {bind }}=\Delta G_{\text {bind }}^{\text {mut }}-\Delta G_{\text {bind }}^{\mathrm{wt}}
$$

We leveraged rapid calculations based on statistical potentials to compute the ensemble-averaged binding free energy changes using equilibrium samples from $\mathrm{MD}$ trajectories. The binding free energy changes were computed by averaging the results over 1000 equilibrium samples for each of the studied systems. The quality and robustness of calculations are assessed in terms of standard errors of the mean based on the five independent trajectories and independent averaging over 500, 1000, and 2000 samples from each trajectory.

\section{ASSOCIATED CONTENT}

\section{(s) Supporting Information}

The Supporting Information is available free of charge at https://pubs.acs.org/doi/10.1021/acsomega.1c03558.

Domains in the full-length SARS-CoV-2 S protein and a detailed structural organization of the $\mathrm{S}-\mathrm{RBD}$, cysteine residues that form disulfide linkages in the $\mathrm{S}$-RBD protein, structural organization of cysteine clusters in the S2 subdomain of the SARS-CoV-2 spike prefusion structure, covariance matrices of residue fluctuations in the S-RBD complexes with nanobodies, and structural mapping of protein stability hotspots for the panel of studied nanobodies (PDF)

\section{AUTHOR INFORMATION}

\section{Corresponding Author}

Gennady M. Verkhivker - Keck Center for Science and Engineering, Schmid College of Science and Technology, Chapman University, Orange, California 92866, United States; Department of Biomedical and Pharmaceutical Sciences, Chapman University School of Pharmacy, Irvine, California 92618, United States; O orcid.org/0000-00024507-4471; Email: verkhivk@chapman.edu

\section{Authors}

Steve Agajanian - Keck Center for Science and Engineering, Schmid College of Science and Technology, Chapman University, Orange, California 92866, United States

Deniz Yasar Oztas - Keck Center for Science and Engineering, Schmid College of Science and Technology, Chapman University, Orange, California 92866, United States

Grace Gupta - Keck Center for Science and Engineering, Schmid College of Science and Technology, Chapman University, Orange, California 92866, United States

Complete contact information is available at:

https://pubs.acs.org/10.1021/acsomega.1c03558

\section{Notes}

The authors declare no competing financial interest.

\section{ACKNOWLEDGMENTS}

This work was supported by the Kay Family Foundation grant A20-0032.

\section{ABBREVIATIONS}

SARS, severe acute respiratory syndrome; ACE2, angiotensinconverting enzyme 2; NTD, N-terminal domain; RBD, receptor-binding domain; CTD1, C-terminal domain 1; CTD2, C-terminal domain 2; FP, fusion peptide; FPPR, fusion peptide proximal region; HR1, heptad repeat $1 ; \mathrm{CH}$, central helix region; $C D$, connector domain; HR2, heptad repeat 2; TM, transmembrane anchor; CT, cytoplasmic tail

\section{REFERENCES}

(1) Tai, W.; He, L.; Zhang, X.; Pu, J.; Voronin, D.; Jiang, S.; Zhou, Y.; Du, L. Characterization of the receptor-binding domain (RBD) of 2019 novel coronavirus: implication for development of RBD protein as a viral attachment inhibitor and vaccine. Cell. Mol. Immunol. 2020, 17, 613-620.

(2) Wang, Q.; Zhang, Y.; Wu, L.; Niu, S.; Song, C.; Zhang, Z.; Lu, G.; Qiao, C.; Hu, Y.; Yuen, K.-Y.; Wang, Q.; Zhou, H.; Yan, J.; Qi, J. 
Structural and functional basis of SARS-CoV-2 entry by using human ACE2. Cell 2020, 181, 894-904.

(3) Wan, Y.; Shang, J.; Graham, R.; Baric, R. S.; Li, F. Receptor recognition by the novel coronavirus from Wuhan: An analysis based on decade-long structural studies of SARS coronavirus. J. Virol. 2020, 94, No. e00127.

(4) Walls, A. C.; Park, Y.-J.; Tortorici, M. A.; Wall, A.; McGuire, A. T.; Veesler, D. Structure, Function, and Antigenicity of the SARSCoV-2 Spike Glycoprotein. Cell 2020, 181, 281-292.

(5) Wrapp, D.; Wang, N.; Corbett, K. S.; Goldsmith, J. A.; Hsieh, C.L.; Abiona, O.; Graham, B. S.; McLellan, J. S. Cryo-EM structure of the 2019-nCoV spike in the prefusion conformation. Science 2020, $367,1260-1263$.

(6) Walls, A. C.; Tortorici, M. A.; Snijder, J.; Xiong, X.; Bosch, B.-J.; Rey, F. A.; Veesler, D. Tectonic conformational changes of a coronavirus spike glycoprotein promote membrane fusion. Proc. Natl. Acad. Sci. U.S.A. 2017, 114, 11157-11162.

(7) Fan, X.; Cao, D.; Kong, L.; Zhang, X. Cryo-EM analysis of the post-fusion structure of the SARS-CoV spike glycoprotein. Nat. Commun. 2020, 11, 3618.

(8) Walls, A. C.; Park, Y.-J.; Tortorici, M. A.; Wall, A.; McGuire, A. T.; Veesler, D. Structure, Function, and Antigenicity of the SARSCoV-2 Spike Glycoprotein. Cell 2020, 181, 281-292.

(9) Wrapp, D.; Wang, N.; Corbett, K. S.; Goldsmith, J. A.; Hsieh, C.L.; Abiona, O.; Graham, B. S.; McLellan, J. S. Cryo-EM structure of the 2019-nCoV spike in the prefusion conformation. Science 2020, 367, 1260-1263.

(10) Cai, Y.; Zhang, J.; Xiao, T.; Peng, H.; Sterling, S. M.; Walsh, R. M., Jr.; Rawson, S.; Rits-Volloch, S.; Chen, B. Distinct conformational states of SARS-CoV-2 spike protein. Science 2020, 369, 1586-1592.

(11) Cai, Y.; Zhang, J.; Xiao, T.; Peng, H.; Sterling, S. M.; Walsh, R. M., Jr.; Rawson, S.; Rits-Volloch, S.; Chen, B. Distinct conformational states of SARS-CoV-2 spike protein. Science 2020, 369, 1586-1592.

(12) Hsieh, C.-L.; Goldsmith, J. A.; Schaub, J. M.; DiVenere, A. M.; Kuo, H.-C.; Javanmardi, K.; Le, K. C.; Wrapp, D.; Lee, A. G.; Liu, Y.; Chou, C.-W.; Byrne, P. O.; Hjorth, C. K.; Johnson, N. V.; LudesMeyers, J.; Nguyen, A. W.; Park, J.; Wang, N.; Amengor, D.; Lavinder, J. J.; Ippolito, G. C.; Maynard, J. A.; Finkelstein, I. J.; McLellan, J. S. Structure-based design of prefusion-stabilized SARS-CoV-2 spikes. Science 2020, 369, 1501-1505.

(13) Henderson, R.; Edwards, R. J.; Mansouri, K.; Janowska, K.; Stalls, V.; Gobeil, S. M. C.; Kopp, M.; Li, D.; Parks, R.; Hsu, A. L.; Borgnia, M. J.; Haynes, B. F.; Acharya, P. Controlling the SARS-CoV2 spike glycoprotein conformation. Nat. Struct. Mol. Biol. 2020, 27, 925-933.

(14) McCallum, M.; Walls, A. C.; Bowen, J. E.; Corti, D.; Veesler, D. Structure-guided covalent stabilization of coronavirus spike glycoprotein trimers in the closed conformation. Nat. Struct. Mol. Biol. 2020, 27, 942-949.

(15) Xiong, X.; Qu, K.; Ciazynska, K. A.; Hosmillo, M.; Carter, A. P.; Ebrahimi, S.; Ke, Z.; Scheres, S. H. W.; Bergamaschi, L.; Grice, G. L.; Zhang, Y.; Bradley, J.; Lyons, P. A.; Smith, K. G. C.; Toshner, M.; Elmer, A.; Ribeiro, C.; Kourampa, J.; Jose, S.; Kennet, J.; Rowlands, J.; Meadows, A.; O’Brien, C.; Rastall, R.; Crucusio, C.; Hewitt, S.; Price, J.; Calder, J.; Canna, L.; Bucke, A.; Tordesillas, H.; Harris, J.; Ruffolo, V.; Domingo, J.; Graves, B.; Butcher, H.; Caputo, D.; Le Gresley, E.; Dunmore, B. J.; Martin, J.; Legchenko, E.; Treacy, C.; Huang, C.; Wood, J.; Sutcliffe, R.; Hodgson, J.; Shih, J.; Graf, S.; Tong, Z.; Mescia, F.; Tilly, T.; O’Donnell, C.; Hunter, K.; Pointon, L.; Pond, N.; Wylot, M.; Jones, E.; Fawke, S.; Bullman, B.; Bergamaschi, L.; Turner, L.; Jarvis, I.; Omarjee, O.; De Sa, A.; Marsden, J.; Betancourt, A.; Perera, M.; Epping, M.; Richoz, N.; Bower, G.; Sharma, R.; Nice, F.; Huhn, O.; Stark, H.; Walker, N.; Stirrups, K.; Ovington, N.; Dewhust, E.; Li, E.; Papadia, S.; Nathan, J. A.; Baker, S.; James, L. C.; Baxendale, H. E.; Goodfellow, I.; Doffinger, R.; Briggs, J. A. G. A thermostable, closed SARS-CoV-2 spike protein trimer. Nat. Struct. Mol. Biol. 2020, 27, 934-941.

(16) Turoňová, B.; Sikora, M.; Schürmann, C.; Hagen, W. J. H.; Welsch, S.; Blanc, F. E. C.; von Bülow, S.; Gecht, M.; Bagola, K.;
Hörner, C.; van Zandbergen, G.; Landry, J.; de Azevedo, N. T. D.; Mosalaganti, S.; Schwarz, A.; Covino, R.; Mühlebach, M. D.; Hummer, G.; Krijnse Locker, J.; Beck, M. In situ structural analysis of SARS-CoV-2 spike reveals flexibility mediated by three hinges. Science 2020, 370, 203-208.

(17) Lu, M.; Uchil, P. D.; Li, W.; Zheng, D.; Terry, D. S.; Gorman, J.; Shi, W.; Zhang, B.; Zhou, T.; Ding, S.; Gasser, R.; Prevost, J.; Beaudoin-Bussieres, G.; Anand, S. P.; Laumaea, A.; Grover, J. R.; Lihong, L.; Ho, D. D.; Mascola, J.; Finzi, A.; Kwong, P. D.; Blanchard, S. C.; Mothes, W. Real-time conformational dynamics of SARS-CoV2 spikes on virus particles. Cell Host Microbe 2020, 28, 880-891.

(18) Xu, C.; Wang, Y.; Liu, C.; Zhang, C.; Han, W.; Hong, X.; Wang, Y.; Hong, Q.; Wang, S.; Zhao, Q.; Wang, Y.; Yang, Y.; Chen, K.; Zheng, W.; Kong, L.; Wang, F.; Zuo, Q.; Huang, Z.; Cong, Y. Conformational dynamics of SARS-CoV-2 trimeric spike glycoprotein in complex with receptor ACE2 revealed by cryo-EM. Sci. Adv. 2021, 7, No. eabe5575.

(19) Benton, D. J.; Wrobel, A. G.; Xu, P.; Roustan, C.; Martin, S. R.; Rosenthal, P. B.; Skehel, J. J.; Gamblin, S. J. Receptor binding and priming of the spike protein of SARS-CoV-2 for membrane fusion. Nature 2020, 588, 327-330.

(20) Wang, C.; Li, W.; Drabek, D.; Okba, N. M. A.; van Haperen, R.; Osterhaus, A. D. M. E.; van Kuppeveld, F. J. M.; Haagmans, B. L.; Grosveld, F.; Bosch, B.-J. A human monoclonal antibody blocking SARS-CoV-2 infection. Nat. Commun. 2020, 11, 2251.

(21) Hwang, W. C.; Lin, Y.; Santelli, E.; Sui, J.; Jaroszewski, L.; Stec, B.; Farzan, M.; Marasco, W. A.; Liddington, R. C. Structural basis of neutralization by a human anti-severe acute respiratory syndrome spike protein antibody, 80R. J. Biol. Chem. 2006, 281, 34610-34616.

(22) Yuan, M.; Wu, N. C.; Zhu, X.; Lee, C.-C. D.; So, R. T. Y.; Lv, H.; Mok, C. K. P.; Wilson, I. A. A highly conserved cryptic epitope in the receptor binding domains of SARS-CoV-2 and SARS-CoV. Science 2020, 368, 630-633.

(23) Huo, J.; Zhao, Y.; Ren, J.; Zhou, D.; Duyvesteyn, H. M. E.; Ginn, H. M.; Carrique, L.; Malinauskas, T.; Ruza, R. R.; Shah, P. N. M.; Tan, T. K.; Rijal, P.; Coombes, N.; Bewley, K. R.; Tree, J. A.; Radecke, J.; Paterson, N. G.; Supasa, P.; Mongkolsapaya, J.; Screaton, G. R.; Carroll, M.; Townsend, A.; Fry, E. E.; Owens, R. J.; Stuart, D. I. Neutralization of SARS-CoV-2 by destruction of the prefusion spike. Cell Host Microbe 2020, 28, 445-454.

(24) Brouwer, P. J. M.; Caniels, T. G.; van der Straten, K.; Snitselaar, J. L.; Aldon, Y.; Bangaru, S.; Torres, J. L.; Okba, N. M. A.; Claireaux, M.; Kerster, G.; Bentlage, A. E. H.; van Haaren, M. M.; Guerra, D.; Burger, J. A.; Schermer, E. E.; Verheul, K. D.; van der Velde, N.; van der Kooi, A.; van Schooten, J.; van Breemen, M. J.; Bijl, T. P. L.; Sliepen, K.; Aartse, A.; Derking, R.; Bontjer, I.; Kootstra, N. A.; Wiersinga, W. J.; Vidarsson, G.; Haagmans, B. L.; Ward, A. B.; de Bree, G. J.; Sanders, R. W.; van Gils, M. J. Potent neutralizing antibodies from COVID-19 patients define multiple targets of vulnerability. Science 2020, 369, 643-650.

(25) Lv, Z.; Deng, Y.-Q.; Ye, Q.; Cao, L.; Sun, C.-Y.; Fan, C.; Huang, W.; Sun, S.; Sun, Y.; Zhu, L.; Chen, Q.; Wang, N.; Nie, J.; Cui, Z.; Zhu, D.; Shaw, N.; Li, X.-F.; Li, Q.; Xie, L.; Wang, Y.; Rao, Z.; Qin, C.-F.; Wang, X. Structural basis for neutralization of SARS-CoV2 and SARS-CoV by a potent therapeutic antibody. Science 2020, 369, $1505-1509$

(26) Pinto, D.; Park, Y.-J.; Beltramello, M.; Walls, A. C.; Tortorici, M. A.; Bianchi, S.; Jaconi, S.; Culap, K.; Zatta, F.; De Marco, A.; Peter, A.; Guarino, B.; Spreafico, R.; Cameroni, E.; Case, J. B.; Chen, R. E.; Havenar-Daughton, C.; Snell, G.; Telenti, A.; Virgin, H. W.; Lanzavecchia, A.; Diamond, M. S.; Fink, K.; Veesler, D.; Corti, D. Cross-neutralization of SARS-CoV-2 by a human monoclonal SARSCoV antibody. Nature 2020, 583, 290-295.

(27) Tortorici, M. A.; Beltramello, M.; Lempp, F. A.; Pinto, D.; Dang, H. V.; Rosen, L. E.; McCallum, M.; Bowen, J.; Minola, A.; Jaconi, S.; Zatta, F.; De Marco, A.; Guarino, B.; Bianchi, S.; Lauron, E. J.; Tucker, H.; Zhou, J.; Peter, A.; Havenar-Daughton, C.; Wojcechowskyj, J. A.; Case, J. B.; Chen, R. E.; Kaiser, H.; MontielRuiz, M.; Meury, M.; Czudnochowski, N.; Spreafico, R.; Dillen, J.; Ng, 
C.; Sprugasci, N.; Culap, K.; Benigni, F.; Abdelnabi, R.; Foo, S.-Y. C.; Schmid, M. A.; Cameroni, E.; Riva, A.; Gabrieli, A.; Galli, M.; Pizzuto, M. S.; Neyts, J.; Diamond, M. S.; Virgin, H. W.; Snell, G.; Corti, D.; Fink, K.; Veesler, D. Ultrapotent human antibodies protect against SARS-CoV-2 challenge via multiple mechanisms. Science 2020, 370, 950-957.

(28) Chi, X.; Yan, R.; Zhang, J.; Zhang, G.; Zhang, Y.; Hao, M.; Zhang, Z.; Fan, P.; Dong, Y.; Yang, Y.; Chen, Z.; Guo, Y.; Zhang, J.; Li, Y.; Song, X.; Chen, Y.; Xia, L.; Fu, L.; Hou, L.; Xu, J.; Yu, C.; Li, J.; Zhou, Q.; Chen, W. A neutralizing human antibody binds to the Nterminal domain of the Spike protein of SARS-CoV-2. Science 2020, $369,650-655$.

(29) Gavor, E.; Choong, Y. K.; Er, S. Y.; Sivaraman, H.; Sivaraman, J. Structural Basis of SARS-CoV-2 and SARS-CoV Antibody Interactions. Trends Immunol. 2020, 41, 1006-1022.

(30) Finkelstein, M. T.; Mermelstein, A. G.; Parker Miller, E.; Seth, P. C.; Stancofski, E.-S. D.; Fera, D. Structural Analysis of Neutralizing Epitopes of the SARS-CoV-2 Spike to Guide Therapy and Vaccine Design Strategies. Viruses 2021, 13, 134.

(31) Barnes, C. O.; Jette, C. A.; Abernathy, M. E.; Dam, K.-M. A.; Esswein, S. R.; Gristick, H. B.; Malyutin, A. G.; Sharaf, N. G.; HueyTubman, K. E.; Lee, Y. E.; Robbiani, D. F.; Nussenzweig, M. C.; West, A. P., Jr.; Bjorkman, P. J. SARS-CoV-2 neutralizing antibody structures inform therapeutic strategies. Nature 2020, 588, 682-687.

(32) Hansen, J.; Baum, A.; Pascal, K. E.; Russo, V.; Giordano, S.; Wloga, E.; Fulton, B. O.; Yan, Y.; Koon, K.; Patel, K.; Chung, K. M.; Hermann, A.; Ullman, E.; Cruz, J.; Rafique, A.; Huang, T.; Fairhurst, J.; Libertiny, C.; Malbec, M.; Lee, W.-y.; Welsh, R.; Farr, G.; Pennington, S.; Deshpande, D.; Cheng, J.; Watty, A.; Bouffard, P.; Babb, R.; Levenkova, N.; Chen, C.; Zhang, B.; Romero Hernandez, A.; Saotome, K.; Zhou, Y.; Franklin, M.; Sivapalasingam, S.; Lye, D. C.; Weston, S.; Logue, J.; Haupt, R.; Frieman, M.; Chen, G.; Olson, W.; Murphy, A. J.; Stahl, N.; Yancopoulos, G. D.; Kyratsous, C. A. Studies in humanized mice and convalescent humans yield a SARSCoV-2 antibody cocktail. Science 2020, 369, 1010-1014.

(33) Wu, Y.; Wang, F.; Shen, C.; Peng, W.; Li, D.; Zhao, C.; Li, Z.; Li, S.; Bi, Y.; Yang, Y.; Gong, Y.; Xiao, H.; Fan, Z.; Tan, S.; Wu, G.; Tan, W.; Lu, X.; Fan, C.; Wang, Q.; Liu, Y.; Zhang, C.; Qi, J.; Gao, G. F.; Gao, F.; Liu, L. A noncompeting pair of human neutralizing antibodies block COVID-19 virus binding to its receptor ACE2. Science 2020, 368, 1274-1278.

(34) Shi, R.; Shan, C.; Duan, X.; Chen, Z.; Liu, P.; Song, J.; Song, T.; Bi, X.; Han, C.; Wu, L.; Gao, G.; Hu, X.; Zhang, Y.; Tong, Z.; Huang, W.; Liu, W. J.; Wu, G.; Zhang, B.; Wang, L.; Qi, J.; Feng, H.; Wang, F.-S.; Wang, Q.; Gao, G. F.; Yuan, Z.; Yan, J. A human neutralizing antibody targets the receptor-binding site of SARS-CoV-2. Nature 2020, 584, 120-124.

(35) Ju, B.; Zhang, Q.; Ge, J.; Wang, R.; Sun, J.; Ge, X.; Yu, J.; Shan, S.; Zhou, B.; Song, S.; Tang, X.; Yu, J.; Lan, J.; Yuan, J.; Wang, H.; Zhao, J.; Zhang, S.; Wang, Y.; Shi, X.; Liu, L.; Zhao, J.; Wang, X.; Zhang, Z.; Zhang, L. Human neutralizing antibodies elicited by SARSCoV-2 infection. Nature 2020, 584, 115-119.

(36) Shi, R.; Shan, C.; Duan, X.; Chen, Z.; Liu, P.; Song, J.; Song, T.; Bi, X.; Han, C.; Wu, L.; Gao, G.; Hu, X.; Zhang, Y.; Tong, Z.; Huang, W.; Liu, W. J.; Wu, G.; Zhang, B.; Wang, L.; Qi, J.; Feng, H.; Wang, F.-S.; Wang, Q.; Gao, G. F.; Yuan, Z.; Yan, J. A human neutralizing antibody targets the receptor-binding site of SARS-CoV-2. Nature 2020, 584, 120-124.

(37) Du, S.; Cao, Y.; Zhu, Q.; Yu, P.; Qi, F.; Wang, G.; Du, X.; Bao, L.; Deng, W.; Zhu, H.; Liu, J.; Nie, J.; Zheng, Y.; Liang, H.; Liu, R.; Gong, S.; Xu, H.; Yisimayi, A.; Lv, Q.; Wang, B.; He, R.; Han, Y.; Zhao, W.; Bai, Y.; Qu, Y.; Gao, X.; Ji, C.; Wang, Q.; Gao, N.; Huang, W.; Wang, Y.; Xie, X. S.; Su, X.-d.; Xiao, J.; Qin, C. Structurally Resolved SARS-CoV-2 Antibody Shows High Efficacy in Severely Infected Hamsters and Provides a Potent Cocktail Pairing Strategy. Cell 2020, 183, 1013-1023.

(38) Baum, A.; Fulton, B. O.; Wloga, E.; Copin, R.; Pascal, K. E.; Russo, V.; Giordano, S.; Lanza, K.; Negron, N.; Ni, M.; Wei, Y.; Atwal, G. S.; Murphy, A. J.; Stahl, N.; Yancopoulos, G. D.; Kyratsous,
C. A. Antibody cocktail to SARS-CoV-2 spike protein prevents rapid mutational escape seen with individual antibodies. Science 2020, 369, 1014-1018.

(39) Starr, T. N.; Greaney, A. J.; Hilton, S. K.; Ellis, D.; Crawford, K. H. D.; Dingens, A. S.; Navarro, M. J.; Bowen, J. E.; Tortorici, M. A.; Walls, A. C.; King, N. P.; Veesler, D.; Bloom, J. D. Deep Mutational Scanning of SARS-CoV-2 Receptor Binding Domain Reveals Constraints on Folding and ACE2 Binding. Cell 2020, 182, 12951310.

(40) Yi, C.; Sun, X.; Ye, J.; Ding, L.; Liu, M.; Yang, Z.; Lu, X.; Zhang, Y.; Ma, L.; Gu, W.; Qu, A.; Xu, J.; Shi, Z.; Ling, Z.; Sun, B. Key residues of the receptor binding motif in the spike protein of SARS$\mathrm{CoV}-2$ that interact with ACE2 and neutralizing antibodies. Cell. Mol. Immunol. 2020, 17, 621-630.

(41) Greaney, A. J.; Starr, T. N.; Gilchuk, P.; Zost, S. J.; Binshtein, E.; Loes, A. N.; Hilton, S. K.; Huddleston, J.; Eguia, R.; Crawford, K. H. D.; Dingens, A. S.; Nargi, R. S.; Sutton, R. E.; Suryadevara, N.; Rothlauf, P. W.; Liu, Z.; Whelan, S. P. J.; Carnahan, R. H.; Crowe, J. E., Jr.; Bloom, J. D. Complete Mapping of Mutations to the SARSCoV-2 Spike Receptor-Binding Domain that Escape Antibody Recognition. Cell Host Microbe 2021, 29, 44-57.

(42) Greaney, A. J.; Loes, A. N.; Crawford, K. H. D.; Starr, T. N.; Malone, K. D.; Chu, H. Y.; Bloom, J. D. Comprehensive mapping of mutations in the SARS-CoV-2 receptor-binding domain that affect recognition by polyclonal human plasma antibodies. Cell Host Microbe 2021, 29, 463-476.

(43) Harvey, W. T.; Carabelli, A. M.; Jackson, B.; Gupta, R. K.; Thomson, E. C.; Harrison, E. M.; Ludden, C.; Reeve, R.; Rambaut, A.; COVID-19 Genomics UK (COG-UK) Consortium; Peacock, S. J.; Robertson, D. L. SARS-CoV-2 variants, spike mutations and immune escape. Nat. Rev. Microbiol. 2021, 19, 409-424.

(44) Fiorentini, S.; Messali, S.; Zani, A.; Caccuri, F.; Giovanetti, M.; Ciccozzi, M.; Caruso, A. First detection of SARS-CoV-2 spike protein N501 mutation in Italy in August, 2020. Lancet Infect. Dis. 2021, 21, No. e147.

(45) Volz, E.; Mishra, S.; Mishra, S.; Chand, M.; Barrett, J. C.; Johnson, R.; Geidelberg, L.; Hinsley, W. R.; Laydon, D. J.; Dabrera, G.; O’Toole, A.; Amato, R.; Ragonnet-Cronin, M.; Harrison, I.; Jackson, B.; Ariani, C. V.; Boyd, O.; Loman, N. J.; McCrone, J. T.; Gonçalves, S.; Jorgensen, D.; Myers, R.; Hill, V.; Jackson, D. K.; Gaythorpe, K.; Groves, N.; Sillitoe, J.; Kwiatkowski, D. P.; Flaxman, S.; Ratmann, O.; Bhatt, S.; Hopkins, S.; Gandy, A.; Rambaut, A.; Ferguson, N. M. Assessing transmissibility of SARS-CoV-2 lineage B.1.1.7 in England. Nature 2021, 593, 266-269.

(46) Davies, N. G.; Jarvis, C. I.; Jarvis, C. I.; Edmunds, W. J.; Jewell, N. P.; Diaz-Ordaz, K.; Keogh, R. H. Increased mortality in community-tested cases of SARS-CoV-2 lineage B.1.1.7. Nature 2021, 593, 270-274.

(47) Tegally, H.; Wilkinson, E.; Giovanetti, M.; Iranzadeh, A.; Fonseca, V.; Giandhari, J.; Doolabh, D.; Pillay, S.; San, E. J.; Msomi, N.; Mlisana, K.; von Gottberg, A.; Walaza, S.; Allam, M.; Ismail, A.; Mohale, T.; Glass, A. J.; Engelbrecht, S.; Van Zyl, G.; Preiser, W.; Petruccione, F.; Sigal, A.; Hardie, D.; Marais, G.; Hsiao, N.-y.; Korsman, S.; Davies, M.-A.; Tyers, L.; Mudau, I.; York, D.; Maslo, C.; Goedhals, D.; Abrahams, S.; Laguda-Akingba, O.; AlisoltaniDehkordi, A.; Godzik, A.; Wibmer, C. K.; Sewell, B. T.; Lourenço, J.; Alcantara, L. C. J.; Kosakovsky Pond, S. L.; Weaver, S.; Martin, D.; Lessells, R. J.; Bhiman, J. N.; Williamson, C.; de Oliveira, T. Detection of a SARS-CoV-2 variant of concern in South Africa. Nature 2021, 592, 438-443.

(48) Tegally, H.; Wilkinson, E.; Lessells, R. J.; Giandhari, J.; Pillay, S.; Msomi, N.; Mlisana, K.; Bhiman, J. N.; von Gottberg, A.; Walaza, S.; Fonseca, V.; Allam, M.; Ismail, A.; Glass, A. J.; Engelbrecht, S.; Van Zyl, G.; Preiser, W.; Williamson, C.; Petruccione, F.; Sigal, A.; Gazy, I.; Hardie, D.; Hsiao, N.-y.; Martin, D.; York, D.; Goedhals, D.; San, E. J.; Giovanetti, M.; Lourenço, J.; Alcantara, L. C. J.; de Oliveira, T. Sixteen novel lineages of SARS-CoV-2 in South Africa. Nat. Med. 2021, 27, 440-446. 
(49) Moyo-Gwete, T.; Madzivhandila, M.; Makhado, Z.; Ayres, F.; Mhlanga, D.; Oosthuysen, B.; Lambson, B. E.; Kgagudi, P.; Tegally, H.; Iranzadeh, A.; Doolabh, D.; Tyers, L.; Chinhoyi, L. R.; Mennen, M.; Skelem, S.; Marais, G.; Wibmer, C. K.; Bhiman, J. N.; Ueckermann, V.; Rossouw, T.; Boswell, M.; de Oliveira, T.; Williamson, C.; Burgers, W. A.; Ntusi, N.; Morris, L.; Moore, P. L. Cross-Reactive Neutralizing Antibody Responses Elicited by SARSCoV-2 501Y.V2 (B.1.351). N. Engl. J. Med. 2021, 384, 2161-2163.

(50) Planas, D.; Bruel, T.; Grzelak, L.; Guivel-Benhassine, F.; Staropoli, I.; Porrot, F.; Planchais, C.; Buchrieser, J.; Rajah, M. M.; Bishop, E.; Albert, M.; Donati, F.; Prot, M.; Behillil, S.; Enouf, V.; Maquart, M.; Smati-Lafarge, M.; Varon, E.; Schortgen, F.; Yahyaoui, L.; Gonzalez, M.; De Sèze, J.; Péré, H.; Veyer, D.; Sève, A.; SimonLorière, E.; Fafi-Kremer, S.; Stefic, K.; Mouquet, H.; Hocqueloux, L.; van der Werf, S.; Prazuck, T.; Schwartz, O. Sensitivity of infectious SARS-CoV-2 B.1.1.7 and B.1.351 variants to neutralizing antibodies. Nat. Med. 2021, 27, 917-924.

(51) Voloch, C. M.; da Silva Francisco, R., Jr.; de Almeida, L. G. P.; Cardoso, C. C.; Brustolini, O. J.; Gerber, A. L.; Guimarães, A. P. C.; Mariani, D.; da Costa, R. M.; Ferreira, O. C., Jr.; Frauches, T. S.; de Mello, C. M. B.; Leitão, I. C.; Galliez, R. M.; Faffe, D. S.; Castiñeiras, T.; Tanuri, A.; de Vasconcelos, A. T. R. Genomic characterization of a novel SARS-CoV-2 lineage from Rio de Janeiro, Brazil. J. Virol. 2021, 95, e00119-e00121.

(52) Sabino, E. C.; Buss, L. F.; Carvalho, M. P. S.; Prete, C. A., Jr.; Crispim, M. A. E.; Fraiji, N. A.; Pereira, R. H. M.; Parag, K. V.; da Silva Peixoto, P.; Kraemer, M. U. G.; Oikawa, M. K.; Salomon, T.; Cucunuba, Z. M.; Castro, M. C.; de Souza Santos, A. A.; Nascimento, V. H.; Pereira, H. S.; Ferguson, N. M.; Pybus, O. G.; Kucharski, A.; Busch, M. P.; Dye, C.; Faria, N. R. Resurgence of COVID-19 in Manaus, Brazil, despite high seroprevalence. Lancet 2021, 397, 452455.

(53) Campbell, F.; Archer, B.; Laurenson-Schafer, H.; Jinnai, Y.; Konings, F.; Batra, N.; Pavlin, B.; Vandemaele, K.; Van Kerkhove, M. D.; Jombart, T.; Morgan, O.; le Polain de Waroux, O. Increased transmissibility and global spread of SARS-CoV-2 variants of concern as at June 2021. Eurosurveillance 2021, 26, 2100509.

(54) Chen, R. E.; Winkler, E. S.; Case, J. B.; Aziati, I. D.; Bricker, T. L.; Joshi, A.; Darling, T. L.; Ying, B.; Errico, J. M.; Shrihari, S.; VanBlargan, L. A.; Xie, X.; Gilchuk, P.; Zost, S. J.; Droit, L.; Liu, Z.; Stumpf, S.; Wang, D.; Handley, S. A.; Stine, W. B., Jr.; Shi, P.-Y.; Davis-Gardner, M. E.; Suthar, M. S.; Knight, M. G.; Andino, R.; Chiu, C. Y.; Ellebedy, A. H.; Fremont, D. H.; Whelan, S. P. J.; Crowe, J. E., Jr.; Purcell, L.; Corti, D.; Boon, A. C. M.; Diamond, M. S. In vivo monoclonal antibody efficacy against SARS-CoV-2 variant strains. Nature 2021, 596, 103-108.

(55) Schoof, M.; Faust, B.; Saunders, R. A.; Sangwan, S.; Rezelj, V.; Hoppe, N.; Boone, M.; Billesbølle, C. B.; Puchades, C.; Azumaya, C. M.; Kratochvil, H. T.; Zimanyi, M.; Deshpande, I.; Liang, J.; Dickinson, S.; Nguyen, H. C.; Chio, C. M.; Merz, G. E.; Thompson, M. C.; Diwanji, D.; Schaefer, K.; Anand, A. A.; Dobzinski, N.; Zha, B. S.; Simoneau, C. R.; Leon, K.; White, K. M.; Chio, U. S.; Gupta, M.; Jin, M.; Li, F.; Liu, Y.; Zhang, K.; Bulkley, D.; Sun, M.; Smith, A. M.; Rizo, A. N.; Moss, F.; Brilot, A. F.; Pourmal, S.; Trenker, R.; Pospiech, T.; Gupta, S.; Barsi-Rhyne, B.; Belyy, V.; Barile-Hill, A. W.; Nock, S.; Liu, Y.; Krogan, N. J.; Ralston, C. Y.; Swaney, D. L.; García-Sastre, A.; Ott, M.; Vignuzzi, M.; Manglik, A.; Walter, P.; Manglik, A. An ultrapotent synthetic nanobody neutralizes SARS-CoV-2 by stabilizing inactive Spike. Science 2020, 370, 14731479.

(56) Xiang, Y.; Nambulli, S.; Xiao, Z.; Liu, H.; Sang, Z.; Duprex, W. P.; Schneidman-Duhovny, D.; Zhang, C.; Shi, Y. Versatile and multivalent nanobodies efficiently neutralize SARS-CoV-2. Science 2020, 370, 1479-1484.

(57) Sun, D.; Sang, Z.; Kim, Y. J.; Xiang, Y.; Cohen, T.; Belford, A. K.; Huet, A.; Conway, J. F.; Sun, J.; Taylor, D. J.; SchneidmanDuhovny, D.; Zhang, C.; Huang, W.; Shi, Y. Potent neutralizing nanobodies resist convergent circulating variants of SARS-CoV-2 by targeting diverse and conserved epitopes. Nat. Commun. 2021, 12, 4676.

(58) Mast, F. D.; Fridy, P. C.; Ketaren, N. E.; Wang, J.; Jacobs, E. Y.; Olivier, J. P.; Sanyal, T.; Molloy, K. R.; Schmidt, F.; Rutkowska, M.; Weisblum, Y.; Rich, L. M.; Vanderwall, E. R.; Dambrauskas, N.; Vigdorovich, V.; Keegan, S.; Jiler, J. B.; Stein, M. E.; Olinares, P. D. B.; Hatziioannou, T.; Sather, D. N.; Debley, J. S.; Fenyö, D.; Sali, A.; Bieniasz, P. D.; Aitchison, J. D.; Chait, B. T.; Rout, M. P. Nanobody Repertoires for Exposing Vulnerabilities of SARS-CoV-2. bioRxiv [Preprint] 2021, DOI: 10.1101/2021.04.08.438911.

(59) Bracken, C. J.; Lim, S. A.; Lim, S. A.; Solomon, P.; Rettko, N. J.; Nguyen, D. P.; Zha, B. S.; Schaefer, K.; Byrnes, J. R.; Zhou, J.; Lui, I.; Liu, J.; QCRG Structural Biology Consortium; Pance, K.; Zhou, X. X.; Leung, K. K.; Wells, J. A. Bi-paratopic and multivalent VH domains block ACE2 binding and neutralize SARS-CoV-2. Nat. Chem. Biol. 2021, 17, 113-121.

(60) Francino-Urdaniz, I. M.; Steiner, P. J.; Kirby, M. B.; Zhao, F.; Haas, C. M.; Barman, S.; Rhodes, E. R.; Leonard, A. C.; Peng, L.; Sprenger, K. G.; Jardine, J. G.; Whitehead, T. A. One-shot identification of SARS-CoV-2 S RBD escape mutants using yeast screening. Cell Rep. 2021, 36, 109627.

(61) Koenig, P.-A.; Das, H.; Liu, H.; Kümmerer, B. M.; Gohr, F. N.; Jenster, L.-M.; Schiffelers, L. D. J.; Tesfamariam, Y. M.; Uchima, M.; Wuerth, J. D.; Gatterdam, K.; Ruetalo, N.; Christensen, M. H.; Fandrey, C. I.; Normann, S.; Tödtmann, J. M. P.; Pritzl, S.; Hanke, L.; Boos, J.; Yuan, M.; Zhu, X.; Schmid-Burgk, J. L.; Kato, H.; Schindler, M.; Wilson, I. A.; Geyer, M.; Ludwig, K. U.; Hällberg, B. M.; Wu, N. C.; Schmidt, F. I. Structure-guided multivalent nanobodies block SARS-CoV-2 infection and suppress mutational escape. Science 2021, 371, No. eabe6230.

(62) Sasisekharan, R. Preparing for the Future - Nanobodies for Covid-19? N. Engl. J. Med. 2021, 384, 1568-1571.

(63) Labroussaa, F.; Jores, J. SARS-CoV-2 nanobodies 2.0. Signal Transduction Targeted Ther. 2021, 6, 202.

(64) Huo, J.; Le Bas, A.; Ruza, R. R.; Duyvesteyn, H. M. E.; Mikolajek, H.; Malinauskas, T.; Tan, T. K.; Rijal, P.; Dumoux, M.; Ward, P. N.; Ren, J.; Zhou, D.; Harrison, P. J.; Weckener, M.; Clare, D. K.; Vogirala, V. K.; Radecke, J.; Moynié, L.; Zhao, Y.; GilbertJaramillo, J.; Knight, M. L.; Tree, J. A.; Buttigieg, K. R.; Coombes, N.; Elmore, M. J.; Carroll, M. W.; Carrique, L.; Shah, P. N. M.; James, W.; Townsend, A. R.; Stuart, D. I.; Owens, R. J.; Naismith, J. H. Neutralizing nanobodies bind SARS-CoV-2 spike RBD and block interaction with ACE2. Nat. Struct. Mol. Biol. 2020, 27, 846-854.

(65) Pymm, P.; Adair, A.; Chan, L.-J.; Cooney, J. P.; Mordant, F. L.; Allison, C. C.; Lopez, E.; Haycroft, E. R.; O’Neill, M. T.; Tan, L. L.; Dietrich, M. H.; Drew, D.; Doerflinger, M.; Dengler, M. A.; Scott, N. E.; Wheatley, A. K.; Gherardin, N. A.; Venugopal, H.; Cromer, D.; Davenport, M. P.; Pickering, R.; Godfrey, D. I.; Purcell, D. F. J.; Kent, S. J.; Chung, A. W.; Subbarao, K.; Pellegrini, M.; Glukhova, A.; Tham, W.-H. Nanobody cocktails potently neutralize SARS-CoV-2 D614G N501Y variant and protect mice. Proc. Natl. Acad. Sci. U.S.A. 2021, 118, No. e2101918118.

(66) Güttler, T.; Aksu, M.; Dickmanns, A.; Stegmann, K. M.; Gregor, K.; Rees, R.; Taxer, W.; Rymarenko, O.; Schünemann, J.; Dienemann, C.; Gunkel, P.; Mussil, B.; Krull, J.; Teichmann, U.; Groß, U.; Cordes, V. C.; Dobbelstein, M.; Görlich, D. Neutralization of SARS-CoV-2 by highly potent, hyperthermostable, and mutationtolerant nanobodies. EMBO J. 2021, 2021, No. e107985.

(67) Xu, J.; Xu, K.; Jung, S.; Conte, A.; Lieberman, J.; Muecksch, F.; Lorenzi, J. C. C.; Park, S.; Schmidt, F.; Wang, Z.; Huang, Y.; Luo, Y.; Nair, M. S.; Wang, P.; Schulz, J. E.; Tessarollo, L.; Bylund, T.; Chuang, G. Y.; Olia, A. S.; Stephens, T.; Teng, I. T.; Tsybovsky, Y.; Zhou, T.; Munster, V.; Ho, D. D.; Hatziioannou, T.; Bieniasz, P. D.; Nussenzweig, M. C.; Kwong, P. D.; Casellas, R. Nanobodies from camelid mice and llamas neutralize SARS-CoV-2 variants. Nature 2021, 595, 278-282.

(68) Gur, M.; Taka, E.; Yilmaz, S. Z.; Kilinc, C.; Aktas, U.; Golcuk, M. Conformational transition of SARS-CoV-2 spike glycoprotein between its closed and open states. J. Chem. Phys. 2020, 153, 075101. 
(69) Woo, H.; Park, S.-J.; Choi, Y. K.; Park, T.; Tanveer, M.; Cao, Y.; Kern, N. R.; Lee, J.; Yeom, M. S.; Croll, T. I.; Seok, C.; Im, W. Developing a Fully Glycosylated Full-Length SARS-CoV-2 Spike Protein Model in a Viral Membrane. J. Phys. Chem. B 2020, 124, $7128-7137$.

(70) Casalino, L.; Gaieb, Z.; Goldsmith, J. A.; Hjorth, C. K.; Dommer, A. C.; Harbison, A. M.; Fogarty, C. A.; Barros, E. P.; Taylor, B. C.; McLellan, J. S.; Fadda, E.; Amaro, R. E. Beyond Shielding: The Roles of Glycans in the SARS-CoV-2 Spike Protein. ACS Cent. Sci. 2020, 6, 1722-1734.

(71) Yu, A.; Pak, A. J.; He, P.; Monje-Galvan, V.; Casalino, L.; Gaieb, Z.; Dommer, A. C.; Amaro, R. E.; Voth, G. A. A multiscale coarse-grained model of the SARS-CoV-2 virion. Biophys. J. 2021, 120, 1097-1104.

(72) Sztain, T.; Ahn, S.-H.; Bogetti, A. T.; Casalino, L.; Goldsmith, J. A.; Seitz, E.; McCool, R. S.; Kearns, F. L.; Acosta-Reyes, F.; Maji, S.; Mashayekhi, G.; McCammon, J. A.; Ourmazd, A.; Frank, J.; McLellan, J. S.; Chong, L. T.; Amaro, R. E. A glycan gate controls opening of the SARS-CoV-2 spike protein. Nat. Chem. 2021, DOI: 10.1038/s41557021-00758-3.

(73) Andreano, E.; Piccini, G.; Licastro, D.; Casalino, L.; Johnson, N. V.; Paciello, I.; Dal Monego, S.; Pantano, E.; Manganaro, N.; Manenti, A.; Manna, R.; Casa, E.; Hyseni, I.; Benincasa, L.; Montomoli, E.; Amaro, R. E.; McLellan, J. S.; Rappuoli, R. SARSCoV-2 escape from a highly neutralizing COVID-19 convalescent plasma. Proc. Natl. Acad. Sci. U.S.A. 2021, 118, No. e2103154118

(74) Sikora, M.; von Bülow, S.; Blanc, F. E. C.; Gecht, M.; Covino, R.; Hummer, G. Computational epitope map of SARS-CoV-2 spike protein. PLoS Comput. Biol. 2021, 17, No. e1008790.

(75) Ghorbani, M.; Brooks, B. R.; Klauda, J. B. Critical Sequence Hotspots for Binding of Novel Coronavirus to Angiotensin Converter Enzyme as Evaluated by Molecular Simulations. J. Phys. Chem. B 2020, 124, 10034-10047.

(76) Taka, E.; Yilmaz, S. Z.; Golcuk, M.; Kilinc, C.; Aktas, U.; Yildiz, A.; Gur, M. Critical Interactions between the SARS-CoV-2 Spike Glycoprotein and the Human ACE2 Receptor. J. Phys. Chem. B 2021, 125, 5537-5548.

(77) Verkhivker, G. Coevolution, dynamics and allostery conspire in shaping cooperative binding and signal transmission of the SARSCoV-2 spike protein with human angiotensin-converting enzyme 2. Int. J. Mol. Sci. 2020, 21, 8268.

(78) Verkhivker, G. M. Molecular simulations and network modeling reveal an allosteric signaling in the SARS-CoV-2 spike proteins. J. Proteome Res. 2020, 19, 4587-4608.

(79) Verkhivker, G. M.; Di Paola, L. Dynamic Network Modeling of Allosteric Interactions and Communication Pathways in the SARSCoV-2 Spike Trimer Mutants: Differential Modulation of Conformational Landscapes and Signal Transmission via Cascades of Regulatory Switches. J. Phys. Chem. B 2021, 125, 850-873.

(80) Verkhivker, G. M.; Di Paola, L. Integrated Biophysical Modeling of the SARS-CoV-2 Spike Protein Binding and Allosteric Interactions with Antibodies. J. Phys. Chem. B 2021, 125, 4596-4619.

(81) Verkhivker, G. M.; Agajanian, S.; Oztas, D. Y.; Gupta, G. Comparative Perturbation-Based Modeling of the SARS-CoV-2 Spike Protein Binding with Host Receptor and Neutralizing Antibodies: Structurally Adaptable Allosteric Communication Hotspots Define Spike Sites Targeted by Global Circulating Mutations. Biochemistry 2021, 60, 1459-1484.

(82) Verkhivker, G.; Agajanian, S.; Oztas, D.; Gupta, G. Dynamic Profiling of Binding and Allosteric Propensities of the SARS-CoV-2 Spike Protein with Different Classes of Antibodies: Mutational and Perturbation-Based Scanning Reveals the Allosteric Duality of Functionally Adaptable Hotspots. J. Chem. Theory Comput. 2021, $17,4578-4598$

(83) Arantes, P. R.; Saha, A.; Palermo, G. Fighting COVID-19 Using Molecular Dynamics Simulations. ACS Cent. Sci. 2020, 6, 1654-1656.

(84) Einav, T.; Yazdi, S.; Coey, A.; Bjorkman, P. J.; Phillips, R. Harnessing Avidity: Quantifying the Entropic and Energetic Effects of
Linker Length and Rigidity for Multivalent Binding of Antibodies to HIV-1. Cell Syst. 2019, 9, 466-474.

(85) Erlendsson, S.; Teilum, K. Binding Revisited-Avidity in Cellular Function and Signaling. Front. Mol. Biosci. 2021, 7, 615565.

(86) Vauquelin, G.; Bricca, G.; Van Liefde, I. Avidity and positive allosteric modulation/cooperativity act hand in hand to increase the residence time of bivalent receptor ligands. Fundam. Clin. Pharmacol. 2014, 28, 530-543.

(87) Guerois, R.; Nielsen, J. E.; Serrano, L. Predicting Changes in the Stability of Proteins and Protein Complexes: A Study of More than 1000 Mutations. J. Mol. Biol. 2002, 320, 369-387.

(88) Tokuriki, N.; Stricher, F.; Schymkowitz, J.; Serrano, L.; Tawfik, D. S. The Stability Effects of Protein Mutations Appear to be Universally Distributed. J. Mol. Biol. 2007, 369, 1318-1332.

(89) Schymkowitz, J.; Borg, J.; Stricher, F.; Nys, R.; Rousseau, F.; Serrano, L. The FoldX Web Server: An Online Force Field. Nucleic Acids Res. 2005, 33, W382-W388.

(90) Van Durme, J.; Delgado, J.; Stricher, F.; Serrano, L.; Schymkowitz, J.; Rousseau, F. A graphical interface for the FoldX forcefield. Bioinformatics 2011, 27, 1711-1712.

(91) Christensen, N. J.; Kepp, K. P. Accurate Stabilities of Laccase Mutants Predicted With a Modified FoldX Protocol. J. Chem. Inf. Model. 2012, 52, 3028-3042.

(92) Christensen, N. J.; Kepp, K. P. Stability Mechanisms of Laccase Isoforms Using a Modified FoldX Protocol Applicable to Widely Different Proteins. J. Chem. Theory Comput. 2013, 9, 3210-3223.

(93) Leander, M.; Yuan, Y.; Meger, A.; Cui, Q.; Raman, S. Functional plasticity and evolutionary adaptation of allosteric regulation. Proc. Natl. Acad. Sci. U.S.A. 2020, 117, 25445-25454.

(94) Dehouck, Y.; Kwasigroch, J. M.; Rooman, M.; Gilis, D. BeAtMuSiC: Prediction of changes in protein-protein binding affinity on mutations. Nucleic Acids Res. 2013, 41, W333-W339.

(95) Dehouck, Y.; Grosfils, A.; Folch, B.; Gilis, D.; Bogaerts, P.; Rooman, M. Fast and accurate predictions of protein stability changes upon mutations using statistical potentials and neural networks: PoPMuSiC-2.0. Bioinformatics 2009, 25, 2537-2543.

(96) Liu, S.; Zhang, C.; Zhou, H.; Zhou, Y. A physical reference state unifies the structure-derived potential of mean force for protein folding and binding. Proteins 2004, 56, 93-101.

(97) Xiong, P.; Zhang, C.; Zheng, W.; Zhang, Y. BindProfX: Assessing Mutation-Induced Binding Affinity Change by Protein Interface Profiles with Pseudo-Counts. J. Mol. Biol. 2017, 429, 426434.

(98) Gonzalez, T. R.; Martin, K. P.; Barnes, J. E.; Patel, J. S.; Ytreberg, F. M. Assessment of software methods for estimating protein-protein relative binding affinities. PLoS One 2020, 15, No. e0240573.

(99) Pucci, F.; Rooman, M. Prediction and evolution of the molecular fitness of SARS-CoV-2 variants: Introducing SpikePro. Viruses 2021, 13, 935.

(100) Berman, H. M.; Westbrook, J.; Feng, Z.; Gilliland, G.; Bhat, T. N.; Weissig, H.; Shindyalov, I. N.; Bourne, P. E. The Protein Data Bank. Nucleic Acids Res. 2000, 28, 235-242.

(101) Rose, P. W.; Prlić, A.; Altunkaya, A.; Bi, C.; Bradley, A. R.; Christie, C. H.; Costanzo, L. D.; Duarte, J. M.; Dutta, S.; Feng, Z.; Green, R. K.; Goodsell, D. S.; Hudson, B.; Kalro, T.; Lowe, R.; Peisach, E.; Randle, C.; Rose, A. S.; Shao, C.; Tao, Y. P.; Valasatava, Y.; Voigt, M.; Westbrook, J. D.; Woo, J.; Yang, H.; Young, J. Y.; Zardecki, C.; Berman, H. M.; Burley, S. K. The RCSB protein data bank: integrative view of protein, gene and 3D structural information. Nucleic Acids Res. 2017, 45, D271-D281.

(102) Hekkelman, M. L.; Te Beek, T. A. H.; Pettifer, S. R.; Thorne, D.; Attwood, T. K.; Vriend, G. WIWS: a protein structure bioinformatics Web service collection. Nucleic Acids Res. 2010, 38, W719-W723.

(103) Fernandez-Fuentes, N.; Zhai, J.; Fiser, A. ArchPRED: A template based loop structure prediction server. Nucleic Acids Res. 2006, 34, W173-W176. 
(104) Krivov, G. G.; Shapovalov, M. V.; Dunbrack, R. L., Jr. Improved prediction of protein side-chain conformations with SCWRL4. Proteins 2009, 77, 778-795.

(105) Bhattacharya, D.; Nowotny, J.; Cao, R.; Cheng, J. 3Drefine: an interactive web server for efficient protein structure refinement. Nucleic Acids Res. 2016, 44, W406-W409.

(106) Watanabe, Y.; Berndsen, Z. T.; Raghwani, J.; Seabright, G. E.; Allen, J. D.; Pybus, O. G.; McLellan, J. S.; Wilson, I. A.; Bowden, T. A.; Ward, A. B.; et al. Vulnerabilities in coronavirus glycan shields despite extensive glycosylation. Nat. Commun. 2020, 11, 2688.

(107) Watanabe, Y.; Allen, J. D.; Wrapp, D.; McLellan, J. S.; Crispin, M. Site-specific glycan analysis of the SARS-CoV-2 spike. Science 2020, 369, 330-333.

(108) Phillips, J. C.; Braun, R.; Wang, W.; Gumbart, J.; Tajkhorshid, E.; Villa, E.; Chipot, C.; Skeel, R. D.; Kalé, L.; Schulten, K. Scalable molecular dynamics with NAMD. J. Comput. Chem. 2005, 26, 17811802.

(109) Best, R. B.; Zhu, X.; Shim, J.; Lopes, P. E. M.; Mittal, J.; Feig, M.; Mackerell, A. D., Jr. Optimization of the Additive CHARMM AllAtom Protein Force Field Targeting Improved Sampling of the Backbone $\phi, \psi$ and Side-Chain $\chi 1$ and $\chi 2$ Dihedral Angles. J. Chem. Theory Comput. 2012, 8, 3257-3273.

(110) Di Pierro, M.; Elber, R.; Leimkuhler, B. A Stochastic Algorithm for the Isobaric-Isothermal Ensemble with Ewald Summations for All Long Range Forces. J. Chem. Theory Comput. 2015, 11, 5624-5637.

(111) Martyna, G. J.; Klein, M. L.; Tuckerman, M. Nosé-Hoover chains: The canonical ensemble via continuous dynamics. J. Chem. Phys. 1992, 97, 2635-2643.

(112) Martyna, G. J.; Tobias, D. J.; Klein, M. L. Constant pressure molecular dynamics algorithms. J. Chem. Phys. 1994, 101, 4177-4189. (113) Koukos, P. I.; Glykos, N. M. Grcarma: A fully automated taskoriented interface for the analysis of molecular dynamics trajectories. J. Comput. Chem. 2013, 34, 2310-2312.

(114) Bahar, I.; Lezon, T. R.; Yang, L.-W.; Eyal, E. Global dynamics of proteins: bridging between structure and function. Annu. Rev. Biophys. 2010, 39, 23-42.

(115) Li, H.; Chang, Y.-Y.; Lee, J. Y.; Bahar, I.; Yang, L.-W. DynOmics: dynamics of structural proteome and beyond. Nucleic Acids Res. 2017, 45, W374-W380.

(116) Eyal, E.; Lum, G.; Bahar, I. The anisotropic network model web server at 2015 (ANM 2.0). Bioinformatics 2015, 31, 1487-1489.

(117) Li, H.; Chang, Y.-Y.; Lee, J. Y.; Bahar, I.; Yang, L.-W. DynOmics: dynamics of structural proteome and beyond. Nucleic Acids Res. 2017, 45, W374-W380. 